\title{
Boussinesq system with measure forcing
}

\author{
Piotr B. Mucha ${ }^{1} \cdot$ Liutang Xue ${ }^{2}$ \\ Received: 13 December 2019 / Revised: 13 August 2020 / Published online: 8 October 2020 \\ (c) The Author(s) 2020
}

\begin{abstract}
The paper analyzes the Navier-Stokes system coupled with the convective-diffusion equation responsible for thermal effects. It is a version of the Boussinesq approximation with a heat source. The problem is studied in the two dimensional plane and the heat source is a measure transported by the flow. For arbitrarily large initial data, we prove global in time existence of unique regular solutions. Measure being a heat source limits regularity of constructed solutions and it requires a non-standard framework of inhomogeneous Besov spaces of the $L^{\infty}\left(0, T ; B_{p, \infty}^{s}\right)$-type. The uniqueness of solutions is proved by using the Lagrangian coordinates.
\end{abstract}

Mathematics Subject Classification Primary 76D03 · 35Q35 · 35Q86

\section{Introduction}

Heat conducting fluids systems are an important part of the fluid mechanics. For the most general models the total energy is usually conserved in time. Viscous fluids generate internal friction and produce thermal effects. Variability of the temperature, on the other hand, creates motion of the fluid. As a basic model the theory considers

\section{Communicated by Y. Giga.}

Piotr B. Mucha

p.mucha@mimuw.edu.pl

Liutang Xue

xuelt@bnu.edu.cn

1 Institute of Applied Mathematics and Mechanics, University of Warsaw, ul. Banacha 2, Warsaw, Poland

2 Laboratory of Mathematics and Complex Systems (MOE), School of Mathematical Sciences, Beijing Normal University, Beijing 100875, People's Republic of China 
the Navier-Stokes-Fourier system for the compressible flows:

$$
\left\{\begin{array}{l}
\partial_{t} \rho+\operatorname{div}(\rho u)=0, \\
\partial_{t}(\rho u)+\operatorname{div}(\rho u \otimes u)-\operatorname{div} S(\theta, \nabla u)+\nabla p(\rho, \theta)=\rho f \\
\partial_{t}(\rho s(\rho, \theta))+\operatorname{div}(\rho s(\rho, \theta) u)+\operatorname{div}\left(\frac{q(\theta, \nabla \theta)}{\theta}\right)=\sigma .
\end{array}\right.
$$

In short, $\rho, u, \theta$ are sought quantities: the density, velocity and temperature of the fluid, respectively. Functions $p(\cdot, \cdot)$ and $s(\cdot, \cdot)$ are the pressure and entropy. The stress tensor $S$ is given in the Newtonian form, the energy flux is given in the Fourier form $q=-\kappa(\theta) \nabla \theta$ and the entropy production $\sigma=\frac{1}{\theta}\left(S: \nabla u+\frac{\kappa(\theta)|\nabla \theta|^{2}}{\theta}\right.$ ) (for more details see [12]).

Nowadays mathematics is able to deliver existence of weak solutions [12-14] for the system (1.1), but regular solutions can be obtained for small data [5,24,29] only. The structure of nonlinearities in (1.1) is complex, hence it is natural to look for a reduced version of the system. Taking a low Mach number limit (see $[9,15]$ ), we obtain an incompressible limit which takes into account weak thermal effects. This system is known as the Boussinesq approximation

$$
\left\{\begin{array}{l}
\partial_{t} \theta+v \cdot \nabla \theta-\Delta \theta=\mu \\
\partial_{t} v+v \cdot \nabla v-\Delta v+\nabla p=\theta e_{d} \\
\operatorname{div} v=0
\end{array}\right.
$$

where $d=2,3, e_{d}$ is the last canonical vector of $\mathbb{R}^{d}, v$ is the velocity and $\theta$ is the temperature. In simple words, the above system (1.2) is the incompressible NavierStokes equations coupled with the heat equation with a drift given by the velocity. Force in the momentum equation is defined by the change of temperature along the direction of the gravitational force (i.e. $e_{d}$-direction). The application of such a system relates to modeling phenomena for which the thermal effects cannot be neglected $[1,11]$. This includes models of atmosphere [21]. As relates to the mathematical characteristics of system (1.2) with $\mu=0$, the well-posedness was studied in [3,4,17,20,28].

It is important to underline the following fact, the system does not preserve the energy. Indeed, the authors in [3] proved that for system (1.2) with $\mu=0$ the norm $\|u(t)\|_{L^{2}}^{2}$ may grow in time. It makes mathematical analysis below more interesting, since the dynamics considered is nontrivial for long time and most of norms of solutions are expected to grow in time.

Let us explain the goal of our paper. We consider a special case of system (1.2) in which $\mu$ is given by a heat source transported by the flow:

$$
\left\{\begin{array}{l}
\partial_{t} \mu+v \cdot \nabla \mu=0, \\
\partial_{t} \theta+v \cdot \nabla \theta-\Delta \theta=\mu, \\
\partial_{t} v+v \cdot \nabla v-\Delta v+\nabla p=\theta e_{d}, \\
\operatorname{div} v=0, \\
\left.(\mu, \theta, v)\right|_{t=0}(x)=\left(\mu_{0}, \theta_{0}, v_{0}\right)(x),
\end{array}\right.
$$


where the system is considered in the whole space $\mathbb{R}^{2}$.

One can think of $\mu$ as describing combustion by a measure like a linear combination of Dirac atoms. From the perspective of physical modeling it would describe the movement of water after putting chemical material, like Sodium $(\mathrm{Na})$, rapidly reacting with water into a pool. We assume that total transfer of energy by $\mu$ is constant in time. We also neglect all other chemical or thermodynamical effects to keep the simple form of system (1.3).

The main goal of the paper is to construct large global-in-time unique solutions. Since the Millennium Problem concerning the regularity of weak solutions to the three dimensional Navier-Stokes system is still open, we thus concentrate on the case of dimension two. The key point is to consider general data admitting initial heat production defined as a Radon measure and large initial data of velocity and temperature. We adopt the framework of Besov spaces $B_{p, \infty}^{s}$ for the initial temperature and velocity in order to be able to apply the maximal regularity results of the heat equation with measure as a force (only $L^{\infty}$-in-time information is available).

Our result is stated as follows.

Theorem 1.1 Let $\mu_{0} \in \mathcal{M}_{+}\left(\mathbb{R}^{2}\right)$ with supp $\mu_{0} \subset B_{R_{0}}(0)$ for some $R_{0}>0$. For each $\sigma \in\left(0, \frac{3}{2}\right]$, let $\theta_{0} \in L^{1} \cap B_{\frac{2}{2-\sigma}, \infty}^{2-\sigma}\left(\mathbb{R}^{2}\right)$ with $\theta_{0} \geq 0$, and $v_{0} \in H^{1}\left(\mathbb{R}^{2}\right)$ be a divergencefree vector field with initial vorticity $\omega_{0}=\partial_{1} v_{2,0}-\partial_{2} v_{1,0} \in B_{\frac{2}{2-\sigma}, \infty}^{3-\sigma}\left(\mathbb{R}^{2}\right)$. Then the system (1.3) admits a global in time unique solution $(\mu, \theta, v)$ such that for any $T>0$,

$$
\mu \in L^{\infty}\left(0, T ; \mathcal{M}_{+}\left(\mathbb{R}^{2}\right)\right) \text {, with } \operatorname{supp} \mu \subset B_{R_{0}+C_{T}},
$$

and

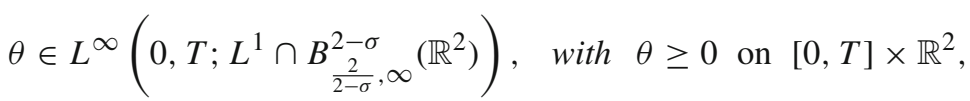

and

$$
\begin{aligned}
& v \in L^{\infty}\left(0, T ; H^{1} \cap W^{1, \infty}\left(\mathbb{R}^{2}\right)\right) \cap L^{2}\left(0, T ; H^{2}\left(\mathbb{R}^{2}\right)\right), \\
& \nabla v \in L^{\infty}\left(0, T ; B_{\frac{2}{2-\sigma}, \infty}^{3-\sigma}\left(\mathbb{R}^{2}\right)\right),
\end{aligned}
$$

where $C_{T}>0$ is a constant depending only on $T$ and the norms of initial data.

The global wellposedness of system (1.3) with smooth forcing $\mu$ in 2D case for large data can be deduced from the current state of the art. Thanks to the classical result of Ladyzhenskaya [22] (and in the language of Besov spaces [10]), we are able to obtain the regular solutions to the Navier-Stokes equations. The system (1.3) from the regularity viewpoint is a weak perturbation of (1.3) 3,4 and basic energy estimates grant the existence at the level of Galerkin's method. However this approach works only for smooth $\mu$. In our case the heat source is a measure and a non-standard approach has to be created. To fit the regularity of temperature and velocity to properties of $\mu$, we apply the Besov spaces of type $B_{p, \infty}^{s}$, defined in Definition (2.3), Sect. 2.2. We also 
assume that the initial heat source is compactly supported in space, to avoid technical problems with the definition of measures at infinity.

Our result has the following interesting ingredients:

* The heat source is a measure which is not vanishing in time-see Proposition 3.1. Solvability of $(1.3)_{1}$ requires high regularity of the velocity (Lipschitz continuity) to guarantee the existence and uniqueness. On the other hand, one can not expect too high regularity of solutions since they are generated by a measure. Thanks to the low spatial dimension, our solutions are regular enough and (1.3) 1 can be solved in terms of characteristics.

* It is an application of inhomogeneous Besov spaces $L^{\infty}\left(0, T ; B_{p, \infty}^{s}\left(\mathbb{R}^{2}\right)\right)$ to address the regularity of solutions. Properties of these spaces allow to consider regularity of $(\mu, \theta, v)$ in the $L^{\infty}$-norm in time, which is required by the basic bound of $\mu$ (1.4). By embedding $\mu$ belongs to $L^{\infty}\left(0, T ; B_{\frac{2}{2-\sigma}, \infty}^{-\sigma}\left(\mathbb{R}^{2}\right)\right)$. This framework fits to the regularity properties of the right-hand side of Eq. (1.3) 2 . In addition, Besov spaces admit the theory of maximal regularity for the heat and Stokes equations, which allows to maintain the full information about the solutions.

* In the construction of the a priori estimates, the source $\mu$ given as a measure does not allow to use the standard bounds by energy norms. The basic $L^{2}$-estimate of velocity $v$ requires the control of $\|\theta(t)\|_{L^{2}}$. However, the properties of $\mu$ imply a natural uniform $L^{1}$-bound $\|\theta\|_{L_{t}^{\infty}\left(L^{1}\right)}$ only. To close the estimation there is a need to interpolate between the $L^{1}$-estimate and a bound in the high-regularity Besov norm. Here the limit case is considered, and the final estimate is obtained by an application of a new logarithmic interpolation inequality for the Besov spaces (Lemma 2.6).

* Since the source $\mu$ is determined by the transport equation, the issue of uniqueness does not fit well to the framework of the Euler coordinates. Our regularity is high enough to define the Lagrangian coordinates (see [7,8] and references therein for this method used in the density-dependent incompressible Navier-Stokes equations). Then after the transformation, $\mu$ becomes fixed in time (see Sect. 3.3). This reduction allows to show uniqueness by elementary energy methods, with the difference of the temperatures in $L^{2}\left(0, T ; L^{2}\left(\mathbb{R}^{2}\right)\right)$.

The simple proof of uniqueness given in this paper requires $\sigma \in(0,3 / 2]$, thus the whole analysis is limited to this case. However by using the idea stated in the following remark it can be extended to $\sigma \in(0,2)$.

Remark 1.2 The restriction $\sigma \in(0,3 / 2]$ comes only from the embedding relation (3.40) used in the uniqueness part, and if we instead use the continuous embeddings that for every $\sigma \in(0,2)$,

$$
\begin{aligned}
B_{\frac{2}{2-\sigma}, \infty}^{2-\sigma}\left(\mathbb{R}^{2}\right) & \hookrightarrow L^{\frac{4}{2-\sigma}}\left(\mathbb{R}^{2}\right) \text { and } L^{\infty}\left(0, T ; L^{2}\left(\mathbb{R}^{2}\right)\right) \cap L^{2}\left(0, T ; \dot{H}^{1}\left(\mathbb{R}^{2}\right)\right) \\
& \hookrightarrow L^{\frac{4}{2-\sigma}}\left(0, T ; L^{\frac{4}{\sigma}}\left(\mathbb{R}^{2}\right)\right),
\end{aligned}
$$

we can similarly show the uniqueness and Theorem 1.1 in the range $\sigma \in(0,2)$.

The outline of this paper is as follows. We present preliminary results including some auxiliary lemmas in Sect. 2. We give the detailed proof of Theorem 1.1 in the whole 
Sect. 3: we firstly show the key a priori estimates of solution $(\mu, \theta, v)$ in Sect. 3.1, then we sketch the proof of existence in Sect. 3.2, and finally we prove the uniqueness by using Lagrangian coordinates in Sect. 3.3. At last in the appendix we show the proof of Lemmas 2.5, 3.4 and 3.5.

The following notation is used throughout this paper.

$\diamond C$ stands for a constant which may be different in different estimates.

$\diamond \mathcal{D}\left(\mathbb{R}^{d}\right)$ or $\mathcal{D}\left(\mathbb{R}^{d} \times[0, T]\right)$ denotes the space of $C^{\infty}$-smooth functions with compact support on $\mathbb{R}^{d}$ or $\mathbb{R}^{d} \times[0, T]$, respectively. $\mathcal{D}^{\prime}\left(\mathbb{R}^{d} \times[0, T]\right)$ is the dual space of $\mathcal{D}\left(\mathbb{R}^{d} \times[0, T]\right)$. Denote $\mathcal{S}\left(\mathbb{R}^{d}\right)$ the Schwartz class of rapidly decreasing $C^{\infty}$-smooth functions, and $\mathcal{S}^{\prime}\left(\mathbb{R}^{d}\right)$ the space of tempered distributions which is the dual space of $\mathcal{S}\left(\mathbb{R}^{d}\right)$.

$\diamond$ For $m \in \mathbb{N}, r \in[1,+\infty], s \in \mathbb{R}$, we denote by $W^{m, r}\left(\mathbb{R}^{d}\right)\left(\dot{W}^{m, r}\left(\mathbb{R}^{d}\right)\right)$ and $H^{s}\left(\mathbb{R}^{d}\right)$ $\left(\dot{H}^{s}\left(\mathbb{R}^{d}\right)\right)$ the usual $L^{r}$-based and $L^{2}$-based inhomogeneous (homogenous) Sobolev spaces. For Banach space $X=X\left(\mathbb{R}^{d}\right)$ and $\rho \in[1, \infty], L^{\rho}(0, T ; X)$ denotes the usual space-time space $L^{\rho}([0, T] ; X)$, which is also abbreviated as $L_{T}^{\rho}(X)$. We also use the usual abbreviation that $\left\|\left(f_{1}, \ldots, f_{n}\right)\right\|_{X}:=\left\|f_{1}\right\|_{X}+\cdots+\left\|f_{n}\right\|_{X}$.

$\diamond$ We use $B_{r}\left(x_{0}\right):=\left\{x \in \mathbb{R}^{d}:\left|x-x_{0}\right|<r\right\}$ to denote the open ball of $\mathbb{R}^{d}$.

\section{Preliminaries}

In this section, we compile basic results related to measure and Lagrangian coordinates, and also show several auxiliary lemmas used in the paper.

\subsection{Results related to measures}

We denote $\mathcal{M}=\mathcal{M}\left(\mathbb{R}^{d}\right)$ as the space of finite Radon measures defined on $\mathbb{R}^{d}$ with total variation topology, i.e., for any $\mu$ Radon measure, define

$$
\|\mu\|_{\mathcal{M}\left(\mathbb{R}^{d}\right)}=|\mu|\left(\mathbb{R}^{d}\right):=\sup \left\{\left|\int_{\mathbb{R}^{d}} f \mathrm{~d} \mu\right|:\|f\|_{L^{\infty}} \leq 1, f \in C_{0}\left(\mathbb{R}^{d}\right)\right\} .
$$

As a consequence of Riesz representation theorem, $\mathcal{M}\left(\mathbb{R}^{d}\right)$ is the dual space of $C_{0}\left(\mathbb{R}^{d}\right)$, that is, $\mathcal{M}\left(\mathbb{R}^{d}\right)=\left(C_{0}\left(\mathbb{R}^{d}\right)\right)^{*}$. For $\left\{\mu_{n}\right\}_{n \in \mathbb{N}} \subset \mathcal{M}\left(\mathbb{R}^{d}\right)$ and $\mu \in \mathcal{M}\left(\mathbb{R}^{d}\right)$, we say $\mu_{n} \rightarrow \mu$ weakly* if

$$
\lim _{n \rightarrow \infty} \int_{\mathbb{R}^{d}} f \mathrm{~d} \mu_{n}=\int_{\mathbb{R}^{d}} f \mathrm{~d} \mu, \quad \text { for every } f \in C_{0}\left(\mathbb{R}^{d}\right)
$$

We also denote $\mathcal{M}=\mathcal{M}\left(\mathbb{R}^{d}, d\right)$ as the space of finite Radon measures on $\mathbb{R}^{d}$ equipped with bounded Lipschitz distance topology, i.e., for any Radon measures $\mu$ and $v$, define 


$$
\begin{aligned}
d(\mu, \nu) & :=\sup \left\{\left|\int_{\mathbb{R}^{d}} f \mathrm{~d} \mu-\int_{\mathbb{R}^{d}} f \mathrm{~d} \nu\right|:\|f\|_{L^{\infty}} \leq 1 \text { and } \operatorname{Lip}(f)\right. \\
& \left.:=\sup _{x \neq y \in \mathbb{R}^{d}} \frac{|f(x)-f(y)|}{|x-y|} \leq 1\right\} .
\end{aligned}
$$

By $\mathcal{M}_{+}=\mathcal{M}_{+}\left(\mathbb{R}^{d}\right)$ we denote the set of nonnegative finite Radon measures on $\mathbb{R}^{d}$, that is,

$$
\mathcal{M}_{+}:=\left\{\mu \in \mathcal{M}\left(\mathbb{R}^{d}\right): \mu \geq 0\right\}
$$

both with the strong total variation and weak $d(\cdot, \cdot)$ topologies.

Definition 2.1 We say that a sequence $\left\{\mu_{n}\right\} \subset \mathcal{M}\left(\mathbb{R}^{d}\right)$ is tight if for any $\epsilon>0$, there exists a compact set $K_{\epsilon} \subset \subset \mathbb{R}^{d}$ so that

$$
\sup _{n \in \mathbb{N}}\left|\mu_{n}\right|\left(\mathbb{R}^{d} \backslash K_{\epsilon}\right)<\epsilon \text {, where }\left|\mu_{n}\right| \text { is the total variation measure of } \mu_{n} \text {. }
$$

Proposition 2.2 (cf. Theorem 2.7 of [18]) Let $\left\{\mu_{n}\right\}_{n \in \mathbb{N}}$ be a tight sequence in $\mathcal{M}\left(\mathbb{R}^{d}\right)$ and let $\mu \in \mathcal{M}\left(\mathbb{R}^{d}\right)$. Then as $n \rightarrow \infty, \mu_{n} \rightarrow \mu$ weakly $*$ if and only if $d\left(\mu_{n}, \mu\right) \rightarrow 0$ and $\sup _{n \in \mathbb{N}}\left|\mu_{n}\right|\left(\mathbb{R}^{d}\right)<\infty$.

The space $\left(\mathcal{M}_{+}, d\right)$ is a complete metric space.

Proposition 2.3 (cf. Corollary 21 of [25]) Let $\left\{\mu_{n}\right\}_{n \in \mathbb{N}}$ be a sequence bounded in $\mathcal{M}_{+}\left(\mathbb{R}^{d}\right)$ with support contained in a given ball. Then there exists a $\left(\mathcal{M}_{+}, d\right)$ convergent subsequence $\left\{\mu_{n_{k}}\right\}$.

\subsection{Besov spaces and auxiliary lemmas}

We recall definitions of inhomogeneous Besov spaces and their space-time counterparts. One can choose two nonnegative radial functions $\chi, \varphi \in \mathcal{D}\left(\mathbb{R}^{d}\right)$ be supported respectively in the ball $\left\{\xi \in \mathbb{R}^{d}:|\xi| \leq \frac{4}{3}\right\}$ and the annulus $\left\{\xi \in \mathbb{R}^{d}: \frac{3}{4} \leq|\xi| \leq \frac{8}{3}\right\}$ such that $\chi(\xi)+\sum_{j \in \mathbb{N}} \varphi\left(2^{-j} \xi\right)=1$ for every $\xi \in \mathbb{R}^{d}$ (see [6]). For every $f \in S^{\prime}\left(\mathbb{R}^{d}\right)$, we define the nonhomogeneous Littlewood-Paley operators as follows

$$
\Delta_{-1} f:=\chi(D) f ; \quad \Delta_{j} f:=\varphi\left(2^{-j} D\right) f, \quad S_{j} f:=\sum_{-1 \leq k \leq j-1} \Delta_{k} f, \quad \forall j \in \mathbb{N} .
$$

Now for $s \in \mathbb{R},(p, r) \in[1,+\infty]^{2}$, the inhomogeneous Besov space $B_{p, r}^{s}=B_{p, r}^{s}\left(\mathbb{R}^{d}\right)$ is defined as

$$
B_{p, r}^{s}:=\left\{f \in \mathcal{S}^{\prime}\left(\mathbb{R}^{d}\right) ;\|f\|_{B_{p, r}^{s}}:=\left(\sum_{j \geq-1} 2^{j s r}\left\|\Delta_{j} f\right\|_{L^{p}}^{r}\right)^{1 / r}<\infty\right\} .
$$


The space-time Besov space $L^{\rho}\left([0, T], B_{p, r}^{s}\right)$, abbreviated by $L_{T}^{\rho}\left(B_{p, r}^{s}\right)$, is the set of tempered distribution $f$ such that

$$
\|f\|_{L_{T}^{\rho}\left(B_{p, r}^{s}\right)}:=\left(\int_{0}^{T}\|f(t)\|_{B_{p, r}^{s}}^{\rho} d t\right)^{1 / \rho}<\infty .
$$

The Besov spaces nowadays belong to the standard toolbox of analytical techniques. They give precise information about regularity of functions. They can be defined also by the real interpolation between classical Sobolev spaces. The basic properties are shown by the embedding theorem

$$
B_{p, r}^{s}\left(\mathbb{R}^{d}\right) \hookrightarrow C_{0}\left(\mathbb{R}^{d}\right) \text { as } p s>d \quad(\text { or } p s=d \text { for } r=1),
$$

and

$$
B_{p, r}^{s}\left(\mathbb{R}^{d}\right) \hookrightarrow L^{q}\left(\mathbb{R}^{d}\right) \text { as } \frac{1}{p}-\frac{1}{q}<\frac{s}{d} \quad\left(\text { or } \frac{1}{p}-\frac{1}{q}=\frac{s}{d} \text { for } r \leq q\right) .
$$

One can refer to [2] for an introduction to this subject from the viewpoint of applications in PDEs.

The following regularity estimates of the heat equation in the framework of Besov spaces are required by our techniques.

Lemma 2.4 (cf. Theorem 2.2.5 of [6]) Let $s \in \mathbb{R}$ and $p \in[1, \infty]$. Let $T>0$, $u_{0} \in B_{p, \infty}^{s}\left(\mathbb{R}^{d}\right)$, and $f \in L_{T}^{\infty}\left(B_{p, \infty}^{s-2}\left(\mathbb{R}^{d}\right)\right)$. Then the following nonhomogeneous heat equation

$$
\partial_{t} u-\Delta u=f,\left.\quad u\right|_{t=0}(x)=u_{0}(x), \quad x \in \mathbb{R}^{d},
$$

has a unique solution $u \in L_{T}^{\infty}\left(B_{p, \infty}^{s}\right)$ and there exists a constant $C=C(d)$ such that

$$
\|u\|_{L_{T}^{\infty}\left(B_{p, \infty}^{s}\left(\mathbb{R}^{d}\right)\right)} \leq C\left(\left\|u_{0}\right\|_{B_{p, \infty}^{s}\left(\mathbb{R}^{d}\right)}+(1+T)\|f\|_{L_{T}^{\infty}\left(B_{p, \infty}^{s-2}\left(\mathbb{R}^{d}\right)\right)}\right)
$$

In obtaining the a priori estimates of the main theorem, we use the following product estimates in Besov spaces (whose proof is put in the appendix section).

Lemma 2.5 Let $v: \mathbb{R}^{2} \rightarrow \mathbb{R}^{2}$ be a divergence-free vector field and $\theta: \mathbb{R}^{2} \rightarrow \mathbb{R}$ be a scalar function.

(1) Let $s \in(0,1), p \in[1, \infty]$. Then there exists a positive constant $C=C(s)$ such that

$$
\begin{aligned}
& \|v \cdot \nabla \theta\|_{B_{p, \infty}^{-s}\left(\mathbb{R}^{2}\right)} \\
& \quad \leq C\left(\|v\|_{L^{2}\left(\mathbb{R}^{2}\right)}+\|\nabla v\|_{L^{2}\left(\mathbb{R}^{2}\right)}\right)\left(\sup _{k \geq-1} 2^{k(1-s)} \sqrt{k+2}\left\|\Delta_{k} \theta\right\|_{L^{p}\left(\mathbb{R}^{2}\right)}\right) .
\end{aligned}
$$


(2) Let $s \in(0, \infty), p \in[1, \infty]$. Then there exists a positive constant $C=C(s)$ such that

$$
\begin{aligned}
\|v \cdot \nabla \theta\|_{B_{p, \infty}^{s}\left(\mathbb{R}^{2}\right)} & \leq C\left(\|v\|_{L^{2 p}\left(\mathbb{R}^{2}\right)}\|\nabla \theta\|_{B_{2 p, \infty}^{s}\left(\mathbb{R}^{2}\right)}+\|v\|_{B_{2 p, \infty}^{s}\left(\mathbb{R}^{2}\right)}\|\nabla \theta\|_{L^{2 p}\left(\mathbb{R}^{2}\right)}\right) \\
& \leq C\|v\|_{B_{2 p, \infty}^{s}\left(\mathbb{R}^{2}\right)}\|\nabla \theta\|_{B_{2 p, \infty}^{s}\left(\mathbb{R}^{2}\right)} .
\end{aligned}
$$

The following interpolation inequalities are required in the main proof.

Lemma 2.6 Let $\theta: \mathbb{R}^{2} \times[0, T] \rightarrow \mathbb{R}$ be a scalar function.

(1) Let $s \in(0,1), p \in[1, \infty]$, then there is a positive constant $C=C(s, p)$ such that

$$
\begin{aligned}
& \sup _{k \geq-1} 2^{k(1-s)} \sqrt{k+2}\left\|\Delta_{k} \theta\right\|_{L_{T}^{\infty}\left(L^{p}\right)} \\
& \leq C\|\theta\|_{L_{T}^{\infty}\left(L^{1}\right)}^{\frac{1}{4-2 / p}}\|\theta\|_{L_{T}^{\infty}\left(B_{p, \infty}^{2-s}\right)}^{\frac{3-s-2 / p}{4-s-2 / p}} \sqrt{\log \left(e+\frac{\|\theta\|_{L_{T}^{\infty}\left(B_{p, \infty}^{2-s}\right)}}{\|\theta\|_{L_{T}^{\infty}\left(L^{1}\right)}}\right)}+C\|\theta\|_{L_{T}^{\infty}\left(L^{1}\right)} .
\end{aligned}
$$

(2) Let $p \in[1, \infty)$, then there is a positive constant $C=C(p)$ such that

$$
\|\theta\|_{L_{T}^{\infty}\left(L^{2}\right)}^{2} \leq C\|\theta\|_{L_{T}^{\infty}\left(L^{1}\right)}\|\theta\|_{L_{T}^{\infty}\left(B_{p, \infty}^{2 / p}\right)} .
$$

Proof of Lemma 2.6 (1) Let $N \in \mathbb{N} \cap[2, \infty)$ be an integer chosen later, then by using Bernsteins's inequality we have

$$
\begin{aligned}
\sup _{k \geq-1} 2^{k(1-s)} \sqrt{k+2}\left\|\Delta_{k} \theta\right\|_{L_{T}^{\infty}\left(L^{p}\right)} \\
\leq \sup _{-1 \leq k \leq N} 2^{k(1-s)} \sqrt{k+2}\left\|\Delta_{k} \theta\right\|_{L_{T}^{\infty}\left(L^{p}\right)}+\sup _{k \geq N} 2^{k(1-s)} \sqrt{k+2}\left\|\Delta_{k} \theta\right\|_{L_{T}^{\infty}\left(L^{p}\right)} \\
\leq C_{0} \sup _{-1 \leq k \leq N} 2^{k(1-s)} \sqrt{2+k} 2^{k\left(2-\frac{2}{p}\right)}\left\|\Delta_{k} \theta\right\|_{L_{T}^{\infty}\left(L^{1}\right)} \\
\quad+C_{0} \sup _{k \geq N} \sqrt{2+k} 2^{-k} 2^{k(2-s)}\left\|\Delta_{k} \theta\right\|_{L_{T}^{\infty}\left(L^{p}\right)} \\
\leq C_{0} 2^{N\left(3-s-\frac{2}{p}\right)} \sqrt{N}\|\theta\|_{L_{T}^{\infty}\left(L^{1}\right)}+C_{0} 2^{-N} \sqrt{N}\|\theta\|_{L_{T}^{\infty}\left(B_{p, \infty}^{2-s}\right)} .
\end{aligned}
$$

Now we define the constant $N$ as

$$
N:= \begin{cases}2, & \text { if }\|\theta\|_{L_{T}^{\infty}\left(B_{p, \infty}^{2-s}\right)} \leq 2\|\theta\|_{L_{T}^{\infty}\left(L^{1}\right)}, \\ {\left[\frac{1}{4-s-2 / p} \log \left(\frac{\|\theta\|_{L_{T}^{\infty}\left(B_{p, \infty}^{2-s}\right)}}{\|\theta\|_{L_{T}^{\infty}\left(L^{1}\right)}}\right)\right]+1,} & \text { if }\|\theta\|_{L_{T}^{\infty}\left(B_{p, \infty}^{2-s}\right)} \geq 2\|\theta\|_{L_{T}^{\infty}\left(L^{1}\right)},\end{cases}
$$

with notation $[a]$ the integer part of $a \in \mathbb{R}$, then it is clear that the desired inequality (2.10) follows by a direct computation. 
(2) We only consider the case $p \in(2, \infty)$, since the remaining case is standard. Let $K \in \mathbb{N}$ be an integer chosen later, then by a high-low frequency decomposition we get

$$
\begin{aligned}
& \|\theta\|_{L_{T}^{\infty}\left(L^{2}\right)}^{2} \leq \sum_{-1 \leq k \leq K}\left\|\Delta_{k} \theta\right\|_{L_{T}^{\infty}\left(L^{2}\right)}^{2}+\sum_{k \geq K}\left\|\Delta_{k} \theta\right\|_{L_{T}^{\infty}\left(L^{2}\right)}^{2} \\
& \leq C_{0} \sum_{-1 \leq k \leq K} 2^{2 k}\left\|\Delta_{k} \theta\right\|_{L_{T}^{\infty}\left(L^{1}\right)}^{2}+C \sum_{k \geq K}\left\|\Delta_{k} \theta\right\|_{L_{T}^{\infty}\left(L^{1}\right)}^{\frac{p-2}{p-1}}\left\|\Delta_{k} \theta\right\|_{L_{T}^{\infty}\left(L^{p}\right)}^{\frac{p}{p-1}} \\
& \leq C 2^{2 K}\|\theta\|_{L_{T}^{\infty}\left(L^{1}\right)}^{2}+C 2^{-\frac{2 K}{p-1}}\|\theta\|_{L_{T}^{\infty}\left(L^{1}\right)}^{\frac{p-2}{p-1}}\|\theta\|_{L_{T}^{\infty}\left(B_{p, \infty}^{2 / p}\right)}^{\frac{p}{p-1}},
\end{aligned}
$$

which clearly guarantees the desired inequality by optimalizing the constant $K$.

The proof of uniqueness requires the following $L^{2}$-based estimate on the Stokes system.

Lemma 2.7 (cf. Lemma 3 of [8]) Let $R$ be a vector field satisfying $\partial_{t} R \in L^{2}\left(\mathbb{R}^{d} \times\right.$ $(0, T])$ and $\nabla \operatorname{div} R \in L^{2}\left(\mathbb{R}^{d} \times(0, T]\right)$. Then the following system

$$
\begin{cases}\partial_{t} u-\Delta u+\nabla P=f, & \text { in } \mathbb{R}^{d} \times(0, T], \\ \operatorname{div} u=\operatorname{div} R, & \text { in } \mathbb{R}^{d} \times(0, T], \\ \left.u\right|_{t=0}=u_{0}, & \text { on } \mathbb{R}^{d},\end{cases}
$$

admits a unique solution $(u, \nabla P)$ which satisfies that

$$
\begin{aligned}
& \|\nabla u\|_{L_{T}^{\infty}\left(L^{2}\right)}+\left\|\left(\partial_{t} u, \nabla^{2} u, \nabla P\right)\right\|_{L_{T}^{2}\left(L^{2}\right)} \\
& \quad \leq C\left(\left\|\nabla u_{0}\right\|_{L^{2}}+\left\|\left(f, \partial_{t} R\right)\right\|_{L_{T}^{2}\left(L^{2}\right)}+\|\nabla \operatorname{div} R\|_{L_{T}^{2}\left(L^{2}\right)}\right),
\end{aligned}
$$

where $C$ is a positive constant independent of $T$.

\subsection{The Lagrangian coordinates}

The use of Lagrangian coordinates plays a fundamental role in the uniqueness proof. In this subsection we introduce notations and basic results related to the Lagrangian coordinates.

Let $X_{v}(t, y)$ solve the following ordinary differential equation (treating $y$ as a parameter)

$$
\frac{\mathrm{d} X_{v}(t, y)}{\mathrm{d} t}=v\left(t, X_{v}(t, y)\right),\left.\quad X_{v}(t, y)\right|_{t=0}=y,
$$

which directly leads to

$$
X_{v}(t, y)=y+\int_{0}^{t} v\left(\tau, X_{v}(\tau, y)\right) \mathrm{d} \tau
$$


Denoting by $\bar{v}(t, y):=v\left(t, X_{v}(t, y)\right)$, flow equation (2.16) can also be expressed as

$$
X_{v}(t, y)=y+\int_{0}^{t} \bar{v}(\tau, y) \mathrm{d} \tau
$$

We list some basic properties for the Lagrangian change of variables.

Lemma 2.8 Assume that $v$ is a velocity vector field belonging to $L^{1}\left(0, T ; \dot{W}^{1, \infty}\left(\mathbb{R}^{d}\right)\right)$. The following statements hold true.

(1) The system (2.15) has a unique solution $X_{v}(t, y)$ on the time interval $[0, T]$ satisfying $\nabla_{y} X_{v} \in L^{\infty}\left(0, T ; L^{\infty}\right)$ with

$$
\left\|\nabla_{y} X_{v}(t)\right\|_{L^{\infty}\left(\mathbb{R}^{d}\right)} \leq \exp \left\{\int_{0}^{t}\left\|\nabla_{x} v(\tau)\right\|_{L^{\infty}\left(\mathbb{R}^{d}\right)} \mathrm{d} \tau\right\}
$$

and also $\nabla_{y} X_{v}(t, y)=\mathrm{Id}+\int_{0}^{t} \nabla_{y} \bar{v}(\tau, y) \mathrm{d} \tau$.

(2) Let $Y_{v}(t, \cdot)$ be the inverse diffeomorphism of $X_{v}(t, \cdot)$, then $\nabla_{x} Y_{v}(t, x)=$ $\left(\nabla_{y} X_{v}(t, y)\right)^{-1}$ with $x=X_{v}(t, y)$, and if

$$
\int_{0}^{t}\left\|\nabla_{y} \bar{v}(\tau)\right\|_{L^{\infty}\left(\mathbb{R}^{d}\right)} \mathrm{d} \tau \leq \frac{1}{2}
$$

we have

$$
\left|\nabla_{x} Y_{v}(t, x)-\mathrm{Id}\right| \leq 2 \int_{0}^{t}\left|\nabla_{y} \bar{v}(\tau, y)\right| \mathrm{d} \tau
$$

(3) If additionally $\nabla_{x}^{2} v \in L^{1}\left(0, T ; L^{p}\left(\mathbb{R}^{d}\right)\right)$ with some $p \in[1, \infty]$, then for every $t \in[0, T]$,

$$
\left\|\nabla_{y}^{2} X_{v}(t)\right\|_{L^{p}\left(\mathbb{R}^{d}\right)} \leq \exp \left\{3 \int_{0}^{t}\left\|\nabla_{x} v(\tau)\right\|_{L^{\infty}\left(\mathbb{R}^{d}\right)} \mathrm{d} \tau\right\} \int_{0}^{t}\left\|\nabla_{x}^{2} v(\tau)\right\|_{L^{p}\left(\mathbb{R}^{d}\right)} \mathrm{d} \tau .
$$

Proof of Lemma 2.8 The proof of (1) and (2) is standard, and one can refer to [8, Proposition 1] for details. For (3), observing that

$$
\begin{aligned}
\nabla_{y}^{2} X_{v}(t, y)= & \int_{0}^{t} \nabla_{y}^{2} \bar{v}(\tau, y) \mathrm{d} \tau=\int_{0}^{t} \nabla_{y} X_{v} \cdot \nabla_{x}^{2} v\left(\tau, X_{v_{1}}\right) \cdot \nabla_{y} X_{v} \mathrm{~d} \tau \\
& +\int_{0}^{t} \nabla_{x} v\left(\tau, X_{v}\right) \cdot \nabla_{y}^{2} X_{v}(\tau, y) \mathrm{d} \tau
\end{aligned}
$$

we get

$$
\left\|\nabla_{y}^{2} X_{v}(t)\right\|_{L^{p}} \leq \int_{0}^{t}\left\|\nabla_{y} X_{v}(\tau)\right\|_{L^{\infty}}^{2}\left\|\nabla_{x}^{2} v(\tau)\right\|_{L^{p}} \mathrm{~d} \tau+\int_{0}^{t}\left\|\nabla_{x} v(\tau)\right\|_{L^{\infty}}\left\|\nabla_{y}^{2} X_{v}(\tau)\right\|_{L^{p}} \mathrm{~d} \tau,
$$


and then, Gronwall's inequality implies

$$
\left\|\nabla^{2} X_{v}(t)\right\|_{L^{p}} \leq e^{\int_{0}^{t}\left\|\nabla_{x} v(\tau)\right\|_{L^{\infty}} \mathrm{d} \tau} \int_{0}^{t}\left\|\nabla X_{v}(\tau)\right\|_{L^{\infty}}^{2}\left\|\nabla_{x}^{2} v(\tau)\right\|_{L^{p}} \mathrm{~d} \tau,
$$

which combined with estimate (2.18) leads to (2.21), as desired.

Under the assumption $v \in L^{1}\left(0, T ; \dot{W}^{1, \infty}\left(\mathbb{R}^{d}\right)\right)$, and using the Lagrangian coordinates introduced as above, we set

$$
\bar{\mu}(t, y):=\mu\left(t, X_{v}(t, y)\right), \quad \bar{\theta}(t, y):=\theta\left(t, X_{v}(t, y)\right), \quad \bar{p}(t, y):=p\left(t, X_{v}(t,(2), 2,2)\right.
$$

then according to properties of the Lagrangian coordinates [7], [8], the Boussinesq type system (1.3) recasts in

$$
\left\{\begin{array}{l}
\partial_{t} \bar{\mu}=0 \\
\partial_{t} \bar{\theta}-\operatorname{div}\left(A_{v} A_{v}^{\mathrm{T}} \nabla_{y} \bar{\theta}\right)=\bar{\mu} \\
\partial_{t} \bar{v}-\operatorname{div}\left(A_{v} A_{v}^{\mathrm{T}} \nabla_{y} \bar{v}\right)+A_{v}^{\mathrm{T}} \nabla_{y} \bar{p}=\bar{\theta} e_{d} \\
\operatorname{div}\left(A_{v} \bar{v}\right)=0, \\
\left.\bar{\mu}\right|_{t=0}=\mu_{0},\left.\quad \bar{\theta}\right|_{t=0}=\theta_{0},\left.\quad \bar{v}\right|_{t=0}=v_{0}
\end{array}\right.
$$

where we adopted the notation $A_{v}(t, y):=\left(\nabla_{y} X_{v}(t, y)\right)^{-1}$ which under the condition (2.19) has the following formula

$$
A_{v}(t, y)=\left(\mathrm{Id}+\left(\nabla_{y} X_{v}-\mathrm{Id}\right)\right)^{-1}=\sum_{k=0}^{\infty}(-1)^{k}\left(\int_{0}^{t} \nabla_{y} \bar{v}(\tau, y) \mathrm{d} \tau\right)^{k} .
$$

As pointed out by [7,8], under the condition (2.19), the system (2.23) in the Lagrangian coordinates is equivalent to the system (1.3) in the Eulerian coordinates.

The first equation of (2.23) implies force $\bar{\mu}$ becomes time independent, hence it is given by the initial datum

$$
\bar{\mu}(t, y) \equiv \mu_{0}(y), \quad \forall t \in[0, T] .
$$

Then system (2.23) reduces to

$$
\left\{\begin{array}{l}
\partial_{t} \bar{\theta}-\Delta \bar{\theta}=\mu_{0}+N_{1}\left(A_{v}, \nabla \bar{\theta}\right), \\
\partial_{t} \bar{v}-\Delta \bar{v}+\nabla \bar{p}=\bar{\theta} e_{d}+N_{2}\left(A_{v}, \nabla \bar{v}\right)+N_{3}\left(A_{v}, \nabla \bar{p}\right), \\
\operatorname{div} \bar{v}=\operatorname{div}\left(\left(\operatorname{Id}-A_{v}\right) \bar{v}\right)=\left(\operatorname{Id}-A_{v}^{\mathrm{T}}\right): \nabla \bar{v}, \\
\left.\bar{\theta}\right|_{t=0}=\theta_{0},\left.\quad \bar{v}\right|_{t=0}=v_{0} .
\end{array}\right.
$$

Nonlinear terms $N_{1}, N_{2}, N_{3}$ are defined by

$$
N_{1}\left(A_{v}, \nabla \bar{\theta}\right):=\operatorname{div}\left(\left(A_{v} A_{v}^{\mathrm{T}}-\mathrm{Id}\right) \nabla \bar{\theta}\right), \quad \text { and }
$$




$$
N_{2}\left(A_{v}, \nabla \bar{v}\right):=\operatorname{div}\left(\left(A_{v} A_{v}^{\mathrm{T}}-\mathrm{Id}\right) \nabla \bar{v}\right), \quad N_{3}\left(A_{v}, \nabla \bar{p}\right):=\left(\mathrm{Id}-A_{v}^{\mathrm{T}}\right) \nabla \bar{p} .
$$

Observe that the left-hand side of (2.26) fits perfectly to the needs of Lemma 2.7. Hence the easiest framework in order to prove the uniqueness property is via the $L^{2}\left(0, T ; L^{2}\left(\mathbb{R}^{2}\right)\right)$ estimate for the difference of temperatures. Note that the change of the Lagrangian coordinates makes our system quasi-linear, and the input from matrix $A_{v}$ is negligible as the time interval is short.

Finally we shall note that the uniqueness to system (1.3) could be proved directly in the Eulerian coordinates. Adopting methods from [19] based on the considerations in Besov spaces with negative regularity index, we shall be able to control the part coming from the transport equation (1.3) $)_{1}$. However this approach for our system seems to be very technical with nontrivial considerations for convection terms.

\section{Proof of Theorem 1.1}

\subsection{A priori estimates}

In this subsection we collect the key a priori estimates of solutions $(\mu, \theta, v)$ to system (1.3).

Proposition 3.1 Let $\mu_{0} \in \mathcal{M}_{+}\left(\mathbb{R}^{2}\right)$ satisfy that $\operatorname{supp} \mu_{0} \subset B_{R_{0}}(0)$ for some $R_{0}>0$. Let $T>0$ be any given, and $(\mu, \theta, v)$ be smooth functions on $\mathbb{R}^{2} \times[0, T]$ solving the system (1.3). Then for every $t \in[0, T]$, we have $\mu(t, x)=\mu_{t}(x) \in \mathcal{M}_{+}\left(\mathbb{R}^{2}\right)$ with

$$
\left\|\mu_{t}\right\|_{\mathcal{M}\left(\mathbb{R}^{2}\right)} \leq\left\|\mu_{0}\right\|_{\mathcal{M}\left(\mathbb{R}^{2}\right)}, \quad \forall t \in[0, T],
$$

and also supp $\mu_{t} \subset B_{R_{0}+C}(0)$ with $C=\|v\|_{L_{T}^{1}\left(L^{\infty}\right)}$.

Proof of Proposition 3.1 Let $X_{v, t}(y)=X_{v}(t, y)$ be the flow function generated by the velocity $v$, which solves Eq. (2.15) or (2.16). Since we assume that $v \in$ $L^{1}\left([0, T] ; W^{1, \infty}\left(\mathbb{R}^{2}\right)\right)$, from Lemma 2.8 , it admits a unique vector field $X_{v, t}: \mathbb{R}^{2} \rightarrow$ $\mathbb{R}^{2}, t \in[0, T]$ which is a diffeomorphism.

Let $Y_{v, t}=Y_{v}(t, \cdot)$ be the inverse diffeomorphism of $X_{v, t}$, then we see that

$$
\mu(t, x)=\mu_{t}(x)=\mu_{0}\left(Y_{v, t}(x)\right) .
$$

Clearly, $\mu_{t} \geq 0$, and since $X_{v, t}$ is volume-preserving (from the divergence-free property of $v$ ), we have 


$$
\begin{aligned}
\left\|\mu_{t}\right\|_{\mathcal{M}\left(\mathbb{R}^{2}\right)}=\sup _{\|g\|_{L^{\infty} \leq 1}}\left|\int_{\mathbb{R}^{2}} g(x) \mathrm{d} \mu_{t}(x)\right| & =\sup _{\|g\|_{L^{\infty} \leq 1}}\left|\int_{\mathbb{R}^{2}} g(x) \mathrm{d} \mu_{0}\left(Y_{v, t}(x)\right)\right| \\
& =\sup _{\|g\|_{L^{\infty} \leq 1}}\left|\int_{\mathbb{R}^{2}} g\left(X_{v, t}(y)\right) \mathrm{d} \mu_{0}(y)\right| \\
& \leq \sup _{\|\tilde{g}\|_{L^{\infty} \leq 1}}\left|\int_{\mathbb{R}^{2}} \tilde{g}(y) \mathrm{d} \mu_{0}(y)\right|=\left\|\mu_{0}\right\|_{\mathcal{M}\left(\mathbb{R}^{2}\right)},
\end{aligned}
$$

where the supremum is taken over all $C_{0}\left(\mathbb{R}^{2}\right)$ functions.

From (3.2) and supp $\mu_{0} \subset B_{R_{0}}(0)$, we get supp $\mu_{t} \subset X_{t}\left(B_{R_{0}}(0)\right)$, and thus formula (2.16) ensures that supp $\mu_{t} \subset B_{R_{0}+\|v\|_{L_{T}^{1}\left(L^{\infty}\right)}}(0)$.

Proposition 3.2 Let $\mu_{0} \in \mathcal{M}_{+}\left(\mathbb{R}^{2}\right)$ satisfy supp $\mu_{0} \subset B_{R_{0}}(0)$ for some $R_{0}>0$, and $\theta_{0} \in L^{1}\left(\mathbb{R}^{2}\right)$ be with $\theta_{0} \geq 0$. For $T>0$ any given, assume $(\mu, \theta, v)$ are smooth functions on $\mathbb{R}^{2} \times[0, T]$ solving system (1.3), and also $\theta$ has the point-wise spatial decay. Then we have that $\theta(t) \geq 0$ for every $t \in[0, T]$ and

$$
\sup _{t \in[0, T]}\|\theta(t)\|_{L^{1}\left(\mathbb{R}^{2}\right)} \leq\left\|\theta_{0}\right\|_{L^{1}\left(\mathbb{R}^{2}\right)}+T\left\|\mu_{0}\right\|_{\mathcal{M}\left(\mathbb{R}^{2}\right)}
$$

Proof of Proposition 3.2 We first prove the nonnegativity property of $\theta(t)$. The proof is standard (e.g. see [23]) and it uses a contradiction argument. Denote by $\Omega_{T}:=$ $(0, T] \times \mathbb{R}^{2}$. We define $\tilde{\theta}(t, x)=\theta(t, x) e^{-t}$ and assume that there is a constant $\lambda>0$ so that

$$
\inf _{(t, x) \in \Omega_{T}} \tilde{\theta}(t, x)=-\lambda
$$

Such a constant $\lambda$ exists since we assume $\tilde{\theta}$ is a bounded smooth function. We also infer that there exists some point $\left(t_{*}, x_{*}\right) \in \Omega_{T}$ attaining this infimum. If not, then there exists a sequence of points $\left(t_{n}, x_{n}\right)_{n \in \mathbb{N}}$ becoming unbounded such that $\tilde{\theta}\left(t_{n}, x_{n}\right) \rightarrow-\lambda$ as $n \rightarrow \infty$, which is a contradiction with the assumption that $\tilde{\theta}$ is a smooth function with suitable spatial decay. From the equation of $\tilde{\theta}$, we get

$$
\left(\partial_{t} \tilde{\theta}\right)\left(t_{*}, x_{*}\right)=-\tilde{\theta}\left(t_{*}, x_{*}\right)-(v \cdot \nabla \tilde{\theta})\left(t_{*}, x_{*}\right)+\Delta \tilde{\theta}\left(t_{*}, x_{*}\right)+\mu\left(t_{*}, x_{*}\right) e^{-t} .
$$

Due to that $\tilde{\theta}$ attains the infimum at $\left(t_{*}, x_{*}\right)$, it yields that $(\nabla \tilde{\theta})\left(t_{*}, x_{*}\right)=0$ and $(\Delta \tilde{\theta})\left(t_{*}, x_{*}\right) \geq 0$, and also $\mu\left(t_{*}, x_{*}\right) \geq 0$ from Proposition 3.1, thus we find

$$
\left(\partial_{t} \tilde{\theta}\right)\left(t_{*}, x_{*}\right) \geq-\tilde{\theta}\left(t_{*}, x_{*}\right)=\lambda
$$

But this clearly contradicts with the fact that $\left(t_{*}, x_{*}\right)$ is the infimum point of $\tilde{\theta}$, hence the nonnegativity of $\theta$ for every $t \in[0, T]$ obviously follows. Note that in the above proof the smoothness of $\theta$ is required. So this part works for smooth approximation of solutions (see Sect. 3.2). Passage to the limit saves the nonnegativity of the temperature. 
Next, we show $\theta \in L^{\infty}\left([0, T] ; L^{1}\left(\mathbb{R}^{2}\right)\right)$. Let $\varphi \in \mathcal{D}\left(\mathbb{R}^{2}\right)$ be a test function satisfying $\operatorname{supp} \varphi \subset B_{1}(0), \varphi \equiv 1$ on $B_{1 / 2}(0)$, and $0 \leq \varphi \leq 1$. Set $\varphi_{R}:=\varphi\left(\frac{\dot{R}}{R}\right)$ for every $R>0$. Multiplying both sides of the equation of $\theta$ with $\varphi_{R}$ and integrating on the spatial variable, we obtain

$$
\frac{\mathrm{d}}{\mathrm{d} t} \int_{\mathbb{R}^{2}} \theta(t) \varphi_{R} \mathrm{~d} x+\int_{\mathbb{R}^{2}} v \cdot \nabla \theta \varphi_{R} \mathrm{~d} x-\int_{\mathbb{R}^{2}} \Delta \theta \varphi_{R} \mathrm{~d} x=\int_{\mathbb{R}^{2}} \mu \varphi_{R} \mathrm{~d} x
$$

By viewing the measure $\mu(t)$ as an element in the dual space of $C_{0}\left(\mathbb{R}^{2}\right)$, we deduce that

$$
\int_{\mathbb{R}^{2}} \mu(t, x) \varphi_{R}(x) \mathrm{d} x=\left(\mu(t), \varphi_{R}\right) \leq\|\mu(t)\|_{\mathcal{M}} \leq\left\|\mu_{0}\right\|_{\mathcal{M}}
$$

Thus integrating on the time interval $[0, t](t \in[0, T])$ and using integration by parts, we find

$$
\begin{aligned}
\int_{\mathbb{R}^{2}} \theta(t) \varphi_{R} \mathrm{~d} x \leq & \int_{\mathbb{R}^{2}} \theta_{0} \varphi_{R} \mathrm{~d} x+\frac{1}{R} \int_{0}^{T} \int_{\mathbb{R}^{2}}|v \theta|\left|\nabla \varphi\left(\frac{x}{R}\right)\right| \mathrm{d} x \mathrm{~d} t \\
& +\frac{1}{R} \int_{0}^{T} \int_{\mathbb{R}^{2}}|\nabla \theta|\left|\nabla \varphi\left(\frac{x}{R}\right)\right| \mathrm{d} x \mathrm{~d} t+T\left\|\mu_{0}\right\|_{\mathcal{M}} .
\end{aligned}
$$

Since we assume $\theta, v$ are smooth functions which guarantee that $v, \theta \in L^{2}\left(\mathbb{R}^{2} \times\right.$ $[0, T])$ and $\nabla \theta \in L^{\frac{3}{2}}\left(\mathbb{R}^{2} \times[0, T]\right)$, by passing $R$ to $+\infty$, it yields that

$$
\int_{\mathbb{R}^{2}} \theta(t, x) \mathrm{d} x \leq \int_{\mathbb{R}^{2}} \theta_{0}(x) \mathrm{d} x+T\left\|\mu_{0}\right\|_{\mathcal{M}} .
$$

Hence the desired inequality (3.3) follows from the nonnegativity of $\theta(t)$.

Proposition 3.3 Let $\mu_{0} \in \mathcal{M}_{+}\left(\mathbb{R}^{2}\right)$ satisfy supp $\mu_{0} \subset B_{R_{0}}(0)$ for some $R_{0}>0$. For each $\sigma \in(0,2)$, let $\theta_{0} \in L^{1} \cap B_{\frac{2}{2-\sigma}, \infty}^{2-\sigma}\left(\mathbb{R}^{2}\right)$ be with $\theta_{0} \geq 0$, and $v_{0} \in H^{1}\left(\mathbb{R}^{2}\right)$ be a divergence-free vector field with initial vorticity $\omega_{0}:=\partial_{1} v_{2,0}-\partial_{2} v_{1,0} \in B_{\frac{2}{2-\sigma}}^{3-\sigma}, \infty\left(\mathbb{R}^{2}\right)$. Let $T>0$ be any given, and assume that $(\mu, \theta, v)$ are smooth functions on $\mathbb{R}^{2} \times[0, T]$ solving the system (1.3). Then we have

$$
\|\theta\|_{L_{T}^{\infty}\left(B_{\frac{2}{2-\sigma}, \infty}^{2-\sigma}\left(\mathbb{R}^{2}\right)\right)}+\|v\|_{L_{T}^{\infty}\left(H^{1}\left(\mathbb{R}^{2}\right)\right)}+\|v\|_{L_{T}^{2}\left(H^{2}\left(\mathbb{R}^{2}\right)\right)} \leq C e^{\exp \left(C(1+T)^{8}\right)}
$$

and

$$
\begin{aligned}
& \left.\|\nabla v\|_{L_{T}^{\infty}\left(B_{\frac{2}{2-\sigma}, \infty}^{3-\sigma}\left(\mathbb{R}^{2}\right)\right)}+\|v\|_{L_{T}^{\infty}\left(W^{1, \infty}\left(\mathbb{R}^{2}\right)\right)}+\left\|\left(\nabla p, \partial_{t} v, \nabla^{2} v\right)\right\|_{L_{T}^{\infty}\left(B_{\frac{2}{2-\sigma}}^{2-\infty}\right)}\right) \\
& \quad \leq C e^{\exp \left(C(1+T)^{8}\right)}
\end{aligned}
$$

where $C>0$ depends only on $\sigma$ and the norms of initial data $\left(\mu_{0}, \theta_{0}, v_{0}\right)$. 
Proof of Proposition 3.3 We first consider the energy type estimates of $v$. By taking the scalar product of the velocity field $v$ with its equation in (1.3), we get

$$
\frac{1}{2} \frac{\mathrm{d}}{\mathrm{d} t}\|v(t)\|_{L^{2}}^{2}+\|\nabla v(t)\|_{L^{2}}^{2} \leq\left|\int_{\mathbb{R}^{2}} \theta v_{2}(t, x) \mathrm{d} x\right| \leq\|\theta(t)\|_{L^{1}\left(\mathbb{R}^{2}\right)}\|v(t)\|_{L^{\infty}\left(\mathbb{R}^{2}\right)} .
$$

By using $L^{1}$-estimate (3.3) and the interpolation inequality, we infer that

$$
\frac{1}{2} \frac{\mathrm{d}}{\mathrm{d} t}\|v(t)\|_{L^{2}\left(\mathbb{R}^{2}\right)}^{2}+\|\nabla v(t)\|_{L^{2}\left(\mathbb{R}^{2}\right)}^{2} \leq C(1+t)\|v(t)\|_{L^{2}\left(\mathbb{R}^{2}\right)}^{1 / 2}\left\|\nabla^{2} v\right\|_{L^{2}\left(\mathbb{R}^{2}\right)}^{1 / 2}
$$

where $C>0$ depends on the norms of initial data $\left\|\mu_{0}\right\|_{\mathcal{M}}$ and $\left\|\theta_{0}\right\|_{L^{1}\left(\mathbb{R}^{2}\right)}$. We then consider the equation of vorticity $\omega:=\operatorname{curl} v=\partial_{1} v_{2}-\partial_{2} v_{1}$, which reads as

$$
\partial_{t} \omega+v \cdot \nabla \omega-\Delta \omega=\partial_{1} \theta .
$$

By taking the inner product of the above equation with $\omega$, and using the integration by parts, we derive that

$$
\frac{1}{2} \frac{\mathrm{d}}{\mathrm{d} t}\|\omega(t)\|_{L^{2}}^{2}+\|\nabla \omega(t)\|_{L^{2}}^{2} \leq\left|\int_{\mathbb{R}^{2}} \theta \partial_{1} \omega(t, x) \mathrm{d} x\right| \leq\|\theta(t)\|_{L^{2}}\|\nabla \omega(t)\|_{L^{2}} .
$$

Young's inequality directly leads to

$$
\frac{\mathrm{d}}{\mathrm{d} t}\|\omega(t)\|_{L^{2}\left(\mathbb{R}^{2}\right)}^{2}+\|\nabla \omega(t)\|_{L^{2}\left(\mathbb{R}^{2}\right)}^{2} \leq\|\theta(t)\|_{L^{2}\left(\mathbb{R}^{2}\right)}^{2} .
$$

Noting that $\left\|\nabla^{2} v\right\|_{L^{2}} \leq\|\nabla \omega\|_{L^{2}}$ and $a b \leq \epsilon a^{4}+C_{\epsilon} b^{4 / 3}$ for any $a, b, \epsilon>0$, we combine the above inequality with (3.6) to get

$$
\begin{aligned}
& \frac{\mathrm{d}}{\mathrm{d} t}\left(\|v(t)\|_{L^{2}}^{2}+\|\omega(t)\|_{L^{2}}^{2}\right)+\|\nabla v(t)\|_{L^{2}}^{2}+\frac{1}{2}\|\nabla \omega(t)\|_{L^{2}}^{2} \leq\|\theta(t)\|_{L^{2}\left(\mathbb{R}^{2}\right)}^{2} \\
& \quad+C(1+t)^{\frac{4}{3}}\|v(t)\|_{L^{2}}^{\frac{2}{3}} .
\end{aligned}
$$

In order to control the norm $\|\theta(t)\|_{L^{2}\left(\mathbb{R}^{2}\right)}$, we will use the equation of $\theta$. However the smoothness of $\theta$ is limited by source $\mu$ which is a measure. Observe that

$$
\mu(t) \in \mathcal{M}\left(\mathbb{R}^{2}\right)=\left(C_{0}\left(\mathbb{R}^{2}\right)\right)^{*} \subset B_{\frac{2}{2-\sigma}, \infty}^{-\sigma}\left(\mathbb{R}^{2}\right),
$$

since $B_{2 / \sigma, 1}^{\sigma}\left(\mathbb{R}^{2}\right) \subset C_{0}\left(\mathbb{R}^{2}\right)$ for $\sigma \in(0,2)$. This Besov regularity of $\mu$ guarantees the temperature in $L^{2}\left(\mathbb{R}^{2}\right)$. Indeed, from estimates of the maximal regularity type we expect that $\theta \in L^{\infty}\left(0, T ; B_{\frac{2}{2-\sigma}}^{2-\sigma}, \infty\right)$, and in combination with (3.3) it yields $\theta(t) \in$ $L^{2}\left(\mathbb{R}^{2}\right)$ 
Hence applying Lemma 2.4 to the equation $\partial_{t} \theta-\Delta \theta=-v \cdot \nabla \theta+\mu$, we infer that for every $t \in[0, T]$,

$$
\begin{aligned}
& \left.\|\theta\|_{L^{\infty}\left(0, t ; B^{2-\sigma}\right.}^{\frac{2}{2-\sigma}, \infty}\left(\mathbb{R}^{2}\right)\right) \\
& \quad \leq C_{0}\left(\left\|\theta_{0}\right\|_{B_{\frac{2}{2-\sigma}, \infty}^{2-\sigma}}+(1+t)\|\mu\|_{L_{t}^{\infty}\left(B_{\frac{2}{2-\sigma}, \infty}^{-\sigma}\right)}+(1+t)\|v \cdot \nabla \theta\|_{L_{t}^{\infty}\left(B_{\frac{2}{2-\sigma}, \infty}^{-\sigma}\right)}\right) .
\end{aligned}
$$

Owing to (3.1) and Lemma 2.5, it follows that

$$
\begin{aligned}
\|\theta\|_{L_{t}^{\infty}\left(B_{\frac{2}{2-\sigma}, \infty}^{2-\sigma}\right)} \leq & C_{0}\left(\left\|\theta_{0}\right\|_{B_{\frac{2}{2-\sigma}, \infty}^{2-\sigma}}+(1+t)\left\|\mu_{0}\right\|_{\mathcal{M}}\right) \\
& +C_{0}(1+t)\|(v, \omega)\|_{L_{t}^{\infty}\left(L^{2}\right)}\left(\sup _{k \geq-1} 2^{k(1-\sigma)} \sqrt{2+k}\left\|\Delta_{k} \theta\right\|_{L_{t}^{\infty}\left(L^{\frac{2}{2-\sigma}}\right)}\right) .
\end{aligned}
$$

We first derive a rough estimate of $\|\theta\|_{L_{t}^{\infty}\left(B_{\frac{2}{2-\sigma}, \infty}^{2-\sigma}\right)}$ in terms of $\|(v, \omega)\|_{L_{t}^{\infty}\left(L^{2}\right)}$. By using the interpolation inequality and Young's inequality, it gives that

$$
\begin{aligned}
& \left.\|\theta\|_{L_{t}^{\infty}\left(B^{2-\sigma} \frac{2}{2-\sigma}, \infty\right.}\right)
\end{aligned}
$$

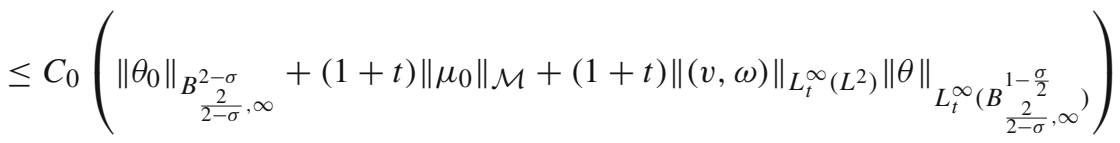

$$
\begin{aligned}
& \leq C_{0}\left(\left\|\theta_{0}\right\|_{\frac{B_{\frac{2}{2-\sigma}, \infty}^{2-\sigma}}{2-\sigma}}+(1+t)\left\|\mu_{0}\right\|_{\mathcal{M}}\right. \\
& \left.+(1+t)\|(v, \omega)\|_{L_{t}^{\infty}\left(L^{2}\right)}\|\theta\|_{L_{t}^{\infty}\left(L^{1}\right)}^{\frac{2-\sigma}{4}}\|\theta\|_{L_{t}^{\infty}\left(B_{\frac{2}{2-\sigma}, \infty}^{2-\sigma}\right)}^{\frac{2+\sigma}{4}}\right) \\
& \leq C(1+t)+C_{0}\left((1+t)^{\frac{4}{2-\sigma}}\|(v, \omega)\|_{L_{t}^{\infty}\left(L^{2}\right)}^{\frac{4}{2-\sigma}}\|\theta\|_{L_{t}^{\infty}\left(L^{1}\right)}\right)+\frac{1}{2}\|\theta\|_{L_{t}^{\infty}\left(B_{\frac{2}{2-\sigma}, \infty}^{2-\sigma}\right)},
\end{aligned}
$$

thus using (3.3) yields

$$
\|\theta\|_{L_{t}^{\infty}\left(B_{\frac{2}{2-\sigma}, \infty}^{2-\sigma}\right)} \leq C(1+t)^{\frac{6-\sigma}{2-\sigma}}\left(1+\|(v, \omega)\|_{L_{t}^{\infty}\left(L^{2}\right)}^{\frac{4}{2-\sigma}}\right),
$$

with $C$ depending on the norms $\left\|\theta_{0}\right\|_{L^{1} \cap B_{\frac{2}{2-\sigma}, \infty}^{2-\sigma}}$ and $\left\|\mu_{0}\right\|_{\mathcal{M}}$.

Then we show a more refined estimate of (3.10) by slightly reducing the power index of $\|(v, \omega)\|_{L_{t}^{\infty} L^{2}}$. Through applying the interpolation inequality (2.10), $L^{1}$-estimate (3.3) and the fact that the function $z \mapsto z^{\frac{1}{2}} \sqrt{\log \left(e+\frac{1}{z}\right)}$ is increasing on $(0, \infty)$, we 
find

$$
\begin{aligned}
\left.\|\theta\|_{L_{t}^{\infty}\left(B_{\frac{2}{2-\sigma}, \infty}^{2-\sigma}\right)}\right) & C_{0}\left(\left\|\theta_{0}\right\|_{B_{\frac{2}{2-\sigma}, \infty}^{2-\sigma}}+(1+t)\left\|\mu_{0}\right\|_{\mathcal{M}}\right) \\
& +C(1+t)\|(v, \omega)\|_{L_{t}^{\infty}\left(L^{2}\right)}\left(\|\theta\|_{L_{t}^{\infty}\left(L^{1}\right)}^{\frac{1}{2}}\|\theta\|_{L_{t}^{\infty}\left(B_{\frac{2}{2-\sigma}, \infty}^{2-\sigma}\right)}^{\frac{1}{2}}\right) \\
\leq & \sqrt{\log \left(e+\frac{\left.\|\theta\|_{L_{t}^{\infty}\left(B_{2 /(2-\sigma), \infty}^{2-\sigma}\right)}\right)}{\left.\|\theta\|_{L_{t}^{\infty}\left(L^{1}\right)}\right)}+\|\theta\|_{L_{t}^{\infty}\left(L^{1}\right)}\right)} \\
& \left(\begin{array}{c}
\left.\|\theta\|_{L_{t}^{\infty}\left(B_{\frac{2}{2-\sigma}}^{\frac{1}{2}}, \infty\right)}\right) \\
\log \left(e+\|\theta\|_{L_{t}^{\infty}\left(B_{\frac{2}{2-\sigma}, \infty}^{2-\sigma}\right)}\right)+1
\end{array}\right),
\end{aligned}
$$

where $C$ depends on the norms of initial data. By virtue of estimate (3.10), we also see that

$$
\begin{aligned}
& \sqrt{\log \left(e+\|\theta\|_{L_{t}^{\infty}\left(B_{\frac{2}{2-\sigma}, \infty}^{2-\sigma}\right)}\right)} \\
& \leq \sqrt{\log \left(\left(e+C(1+t)^{\frac{6-\sigma}{2-\sigma}}\right)\left(e+\|(v, \omega)\|_{L_{t}^{\infty}\left(L^{2}\right)}^{\frac{4}{2-\sigma}}\right)\right)} \\
& \leq \sqrt{1+\log \left(e+C(1+t)^{\frac{6-\sigma}{2-\sigma}}\right)} \sqrt{\log \left(e+\|(v, \omega)\|_{L_{t}^{\infty}\left(L^{2}\right)}^{\frac{4}{2-\sigma}}\right)} \\
& \leq C(1+t) \sqrt{\log \left(e+\|(v, \omega)\|_{L_{t}^{\infty}\left(L^{2}\right)}^{2}\right)}
\end{aligned}
$$

thus inserting this inequality into (3.11) leads to that

$$
\begin{gathered}
\|\theta\|_{L_{t}^{\infty}\left(B_{\frac{2}{2-\sigma}, \infty}^{2-\sigma}\right)} \leq C(1+t)+C(1+t)^{3}\|(v, \omega)\|_{L_{t}^{\infty}\left(L^{2}\right)} \\
\left(\sqrt{\log \left(e+\|(v, \omega)\|_{L_{t}^{\infty}\left(L^{2}\right)}^{2}\right)}\|\theta\|_{L_{t}^{\infty}\left(B_{\frac{2}{2-\sigma}, \infty}^{2-\sigma}\right)}^{\frac{1}{2}}+1\right) .
\end{gathered}
$$


By arguing as (3.9) and (3.10), we obtain

$$
\|\theta\|_{L_{t}^{\infty}\left(B_{\frac{2}{2-\sigma}, \infty}^{2-\sigma}\right)} \leq C(1+t)^{6}\left(1+\|(v, \omega)\|_{L_{t}^{\infty}\left(L^{2}\right)}^{2}\right) \log \left(e+\|(v, \omega)\|_{L_{t}^{\infty}\left(L^{2}\right)}^{2}\right) .
$$

Now we go back to inequality (3.8). By using the interpolation inequality (2.11), estimates (3.3) and (3.12), we deduce that

$$
\begin{aligned}
& \frac{\mathrm{d}}{\mathrm{d} t}\|(v, \omega)(t)\|_{L^{2}}^{2}+\frac{1}{2}\|(\nabla v, \nabla \omega)(t)\|_{L^{2}}^{2} \\
& \quad \leq C\|\theta\|_{L_{t}^{\infty}\left(L^{1}\right)}\|\theta\|_{L_{t}^{\infty}\left(B_{\frac{2}{2-\sigma}, \infty}^{2-\sigma}\right)}+C(1+t)^{\frac{4}{3}}\|v(t)\|_{L^{2}\left(\mathbb{R}^{2}\right)}^{\frac{2}{3}} \\
& \quad \leq C(1+t)^{7}\left(1+\|(v, \omega)\|_{L_{t}^{\infty}\left(L^{2}\right)}^{2} \log \left(e+\|(v, \omega)\|_{L_{t}^{\infty}\left(L^{2}\right)}^{2}\right)\right) .
\end{aligned}
$$

Integrating on the time variable yields that for every $t \in[0, T]$,

$$
\begin{aligned}
& \|(v, \omega)\|_{L_{t}^{\infty}\left(L^{2}\right)}^{2}+\|(\nabla v, \nabla \omega)\|_{L_{t}^{2}\left(L^{2}\right)}^{2} \\
& \leq C\left(\left\|\left(v_{0}, \omega_{0}\right)\right\|_{L^{2}}^{2}+(1+t)^{8}\right) \\
& \quad+C \int_{0}^{t}(1+\tau)^{7}\|(v, \omega)\|_{L_{\tau}^{\infty}\left(L^{2}\right)}^{2} \log \left(e+\|(v, \omega)\|_{L_{\tau}^{\infty}\left(L^{2}\right)}^{2}\right) \mathrm{d} \tau .
\end{aligned}
$$

Grönwall's inequality guarantees that

$$
\|(v, \omega)\|_{L_{T}^{\infty}\left(L^{2}\right)}^{2}+\|(\nabla v, \nabla \omega)\|_{L_{T}^{2}\left(L^{2}\right)}^{2} \leq C e^{\exp \left(C(1+T)^{8}\right)},
$$

where $C$ depends on the norms $\left\|v_{0}\right\|_{H^{1}},\left\|\theta_{0}\right\|_{L^{1} \cap B_{\frac{2}{2-\sigma}, \infty}^{2-\sigma}}$ and $\left\|\mu_{0}\right\|_{\mathcal{M}}$. Plugging the above estimate into (3.10) leads to

$$
\|\theta\|_{L_{t}^{\infty}\left(B_{\frac{2}{2-\sigma}, \infty}^{2-\sigma}\right)} \leq C e^{\exp \left(C(1+T)^{8}\right)}
$$

which combined with (3.14) and the facts $\|\nabla v\|_{L^{2}} \leq\|\omega\|_{L^{2}}$ and $\left\|\nabla^{2} v\right\|_{L^{2}} \leq\|\nabla \omega\|_{L^{2}}$ implies the desired estimate (3.4).

Next we turn to the proof of (3.5). By viewing the equation of $\omega$ (3.7) as a heat equation with forcing, we use estimates (2.7) and (2.9) to get

$$
\begin{aligned}
& \|\omega\|_{L_{T}^{\infty}\left(B_{\frac{2}{2-\sigma}, \infty}^{3-\sigma}\right)} \leq C_{0}\left(\left\|\omega_{0}\right\|_{B_{\frac{2}{2-\sigma}, \infty}^{3-\sigma}\left(\mathbb{R}^{2}\right)}+(1+T)\left\|\partial_{1} \theta\right\|_{L_{T}^{\infty}\left(B_{\frac{2}{2-\sigma}, \infty}^{1-\sigma}\right)}\right) \\
& \left.+(1+T)\|v \cdot \nabla \omega\|_{L_{T}^{\infty}\left(B_{\frac{2}{2-\sigma}, \infty}^{1-\sigma}\right)}\right)
\end{aligned}
$$




$$
\begin{aligned}
\leq & C_{0}\left(\left\|\omega_{0}\right\|_{B_{\frac{2}{2-\sigma}, \infty}^{3-\sigma}}+(1+T)\|\theta\|_{L_{T}^{\infty}\left(B_{\frac{2}{2-\sigma}, \infty}^{2-\sigma}\right)}\right) \\
& \left.+(1+T)\|v\|_{L_{T}^{\infty}\left(B_{\frac{4}{2-\sigma}, \infty}^{1-\sigma}\right)}\|\omega\|_{L_{T}^{\infty}\left(B_{\frac{4}{2-\sigma}, \infty}^{2-\sigma}\right)}\right)
\end{aligned}
$$

In view of (3.14)-(3.15), the continuous embedding $H^{1}\left(\mathbb{R}^{2}\right) \hookrightarrow B_{\frac{4}{2-\sigma}, \infty}^{1-\sigma}\left(\mathbb{R}^{2}\right)$, the

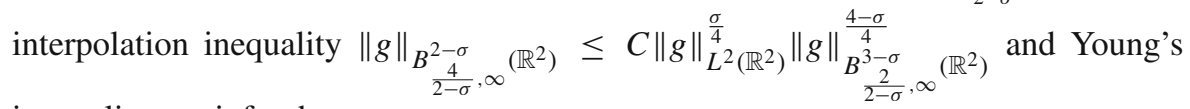
inequality, we infer that

$$
\begin{aligned}
\|\omega\|_{L_{T}^{\infty}\left(B_{\frac{2}{2-\sigma}, \infty}^{3-\sigma}\left(\mathbb{R}^{2}\right)\right)} \leq & C e^{\exp \left(C(1+T)^{8}\right)} \\
& +C_{0}(1+T)\|v\|_{L_{T}^{\infty}\left(H^{1}\right)}\|\omega\|_{L_{T}^{\infty}\left(L^{2}\right)}^{\frac{\sigma}{4}}\|\omega\|_{L_{T}^{\infty}\left(B_{\frac{2}{2-\sigma}, \infty}^{3-\sigma}\left(\mathbb{R}^{2}\right)\right)}^{\frac{4-\sigma}{4}} \\
\leq & C e^{\exp \left(C(1+T)^{8}\right)}+\left(C_{0}(1+T)\|v\|_{L_{T}^{\infty}\left(H^{1}\right)}\right)^{\frac{4}{\sigma}}\|\omega\|_{L_{T}^{\infty}\left(L^{2}\right)} \\
& \left.+\frac{1}{2}\|\omega\|_{L_{T}^{\infty}\left(B_{\frac{2}{2-\sigma}}^{3-\sigma}, \infty\right.}\right) \\
\leq & C e^{\exp \left(C(1+T)^{8}\right)}+\frac{1}{2}\|\omega\|_{L_{T}^{\infty}\left(B_{\frac{2}{2-\sigma}, \infty}^{3-\sigma}\left(\mathbb{R}^{2}\right)\right)}
\end{aligned}
$$

thus the Calderón-Zygmund theorem implies

$$
\|\nabla v\|_{L_{T}^{\infty}\left(B_{\frac{2}{2-\sigma}, \infty}^{3-\sigma}\left(\mathbb{R}^{2}\right)\right)} \leq C\|\omega\|_{L_{T}^{\infty}\left(B_{\frac{2}{2-\sigma}, \infty}^{3-\sigma}\left(\mathbb{R}^{2}\right)\right)} \leq C e^{\exp \left(C(1+T)^{8}\right)}
$$

and also

$$
\left.\left\|\nabla^{2} v\right\|_{L_{T}^{\infty}\left(B_{\frac{2}{2-\sigma}, \infty}^{2-\sigma}\left(\mathbb{R}^{2}\right)\right)} \leq C_{0}\|\nabla v\|_{L_{T}^{\infty}\left(B_{\frac{2}{2-\sigma}, \infty}^{3-\sigma}\right.}\left(\mathbb{R}^{2}\right)\right) \leq C e^{\exp \left(C(1+T)^{8}\right)},
$$

where $C$ depends on the norms $\left\|v_{0}\right\|_{H^{1}},\left\|\omega_{0}\right\|_{\frac{B_{\frac{2}{2-\sigma}, \infty}^{3-\sigma}}{2-\sigma}},\left\|\theta_{0}\right\|_{L^{1} \cap B_{\frac{2}{2-\sigma}, \infty}^{2-\sigma}}$ and $\left\|\mu_{0}\right\|_{\mathcal{M}}$. Besides, by virtue of the high-low frequency decomposition and Bernstein's inequality, we have

$$
\begin{aligned}
\|v\|_{L_{T}^{\infty}\left(W^{1, \infty}\left(\mathbb{R}^{2}\right)\right)} & \leq C_{0}\left\|\Delta_{-1} v\right\|_{L_{T}^{\infty}\left(L^{\infty}\left(\mathbb{R}^{2}\right)\right)}+C_{0} \sum_{q \in \mathbb{N}}\left\|\Delta_{q} \nabla v\right\|_{L_{T}^{\infty}\left(L^{\infty}\left(\mathbb{R}^{2}\right)\right)} \\
& \leq C_{0}\|v\|_{L_{T}^{\infty}\left(L^{2}\left(\mathbb{R}^{2}\right)\right)}+C_{0} \sum_{q \in \mathbb{N}} 2^{-q} 2^{q(3-\sigma)}\left\|\Delta_{q} \nabla v\right\|_{L_{T}^{\infty}\left(L^{2-\sigma}\left(\mathbb{R}^{2}\right)\right)} \\
& \leq C_{0}\|v\|_{L_{T}^{\infty}\left(L^{2}\left(\mathbb{R}^{2}\right)\right)}+C_{0}\|\nabla v\|_{L_{T}^{\infty}\left(B_{\frac{2}{2-\sigma}, \infty}^{3-\sigma}\left(\mathbb{R}^{2}\right)\right)} \leq C e^{\exp \left(C(1+T)^{8}\right)},
\end{aligned}
$$


and similarly,

$\|v\|_{L_{T}^{\infty}\left(B_{\frac{4}{2-\sigma}, \infty}^{2-\sigma}\left(\mathbb{R}^{2}\right)\right)} \leq C\|v\|_{L_{T}^{\infty}\left(L^{2}\left(\mathbb{R}^{2}\right)\right)}+C\|\nabla v\|_{L_{T}^{\infty}\left(B_{\frac{2}{2-\sigma}, \infty}^{3-\sigma}\left(\mathbb{R}^{2}\right)\right)} \leq C e^{\exp \left(C(1+T)^{8}\right)}$

By applying the Eq. (1.3) 3 and the divergence-free condition of $v$, we see that $\nabla p=\nabla(-\Delta)^{-1} \operatorname{div}(v \cdot \nabla v)-\nabla \partial_{1}(-\Delta)^{-1} \theta$, thus in combination with the CalderónZygmund theorem and inequality (2.9), and using embedding $B_{\frac{2}{2-\sigma}, \infty}^{3-\sigma}\left(\mathbb{R}^{2}\right) \hookrightarrow$ $B_{\frac{4}{2-\sigma}, \infty}^{2-\sigma}\left(\mathbb{R}^{2}\right)$, it leads to

$$
\begin{aligned}
\|\nabla p\|_{L_{T}^{\infty}\left(B_{\frac{2}{2-\sigma}, \infty}^{2-\sigma}\left(\mathbb{R}^{2}\right)\right)} & \left.\leq C\|v \cdot \nabla v\|_{L_{T}^{\infty}\left(B_{\frac{2}{2-\sigma}, \infty}^{2-\sigma}\right)}+C\|\theta\|_{L_{T}^{\infty}\left(B_{\frac{2}{2-\sigma}, \infty}^{2-\sigma}\right)}\right) \\
& \left.\leq C\|v\|_{L_{T}^{\infty}\left(B_{\frac{4}{2-\sigma}, \infty}^{2-\sigma}\right)}\|\nabla v\|_{L_{T}^{\infty}\left(B_{\frac{4}{2-\sigma}, \infty}^{2-\sigma}\right)}+C\|\theta\|_{L_{T}^{\infty}\left(B_{\frac{2}{2-\sigma}}^{2-\sigma}, \infty\right)}\right) \\
& \leq C e^{\exp \left(C(1+T)^{8}\right)}
\end{aligned}
$$

Furthermore, Eq. (1.3) 3 and the above estimates also yield that

$$
\left\|\partial_{t} v\right\|_{L_{T}^{\infty}\left(B_{\frac{2}{2-\sigma}, \infty}^{2-\sigma}\left(\mathbb{R}^{2}\right)\right)} \leq C_{0}\|(v \cdot \nabla v, \Delta v, \nabla p, \theta)\|_{L_{T}^{\infty}\left(B_{\frac{2}{2-\sigma}, \infty}^{2-\sigma}\right)} \leq C e^{\exp \left(C(1+T)^{8}\right)} .
$$

\subsection{Global existence}

The issue of existence for our system is not immediate since $\mu=\mu(x, t)$ is merely a measure. In order to construct a suitable approximation we consider the system with smooth initial data. We assume that

$$
\left.\mu^{n}\right|_{t=0},\left.\quad \theta^{n}\right|_{t=0},\left.\quad v^{n}\right|_{t=0} \text { belong to the Schwartz class over the plane, }
$$

where $n \in \mathbb{N}^{+}$is an approximation parameter ( $n \rightarrow \infty$ in the end) and they converge to the initial states $\mu_{0}, \theta_{0}, v_{0}$ in spaces prescribed by Theorem 1.1 (at least in a weak sentence).

To show the existence of system (1.3) with such initial data (3.21), we will use a standard approach via Galerkin method. An approximation we build relies on the following spaces:

$* H^{2}\left(\mathbb{R}^{2}\right)$ for the velocity field in the divergence-free subset

and

* $H^{1}\left(\mathbb{R}^{2}\right)$ for the temperature. 
In short, $v^{n, N}$ and $\theta^{n, N}$ are approximations based on the $N$-dimensional restriction of $H^{2}$ and $H^{1}$ spaces. We have

$$
v^{n, N}=\sum_{k=1}^{N} V_{k}^{n, N}(t) w_{k}(x), \quad \theta^{n, N}=\sum_{k=1}^{N} \Theta_{k}^{n, N}(t) g_{k}(x) .
$$

Vectors $w_{k}$ and $g_{k}$ are the based vectors of $H^{2}\left(\mathbb{R}^{2} ; \mathbb{R}^{2}\right)$ of the divergence-free subspace and $H^{1}\left(\mathbb{R}^{2} ; \mathbb{R}\right)$, respectively. The sought functions $V_{k}^{n, N}(t)$ and $\Theta_{k}^{n, N}(t)$ are derived by solving of the following ODEs

$$
\begin{gathered}
\left(\partial_{t} \theta^{n, N}, \psi^{N}\right)+\left(v^{n, N} \cdot \nabla \theta^{n, N}, \psi^{N}\right)+\left(\nabla \theta^{n, N}, \nabla \psi^{N}\right)=\left(\mu^{n, N}, \psi^{N}\right), \\
\left(\partial_{t} v^{n, N}, \Psi^{N}\right)+\left(v^{n, N} \cdot \nabla v^{n, N}, \Psi^{N}\right)+\left(\nabla v^{n, N}, \nabla \Psi^{N}\right)=\left(\theta^{n, N} e_{2}, \Psi^{N}\right), \\
V_{k}^{n, N}(0)=\left(\phi_{n} * v_{0}, w_{k}\right), \quad \Theta_{k}^{n, N}(0)=\left(\phi_{n} * \theta_{0}, g_{k}\right), \quad k=1, \ldots, N,
\end{gathered}
$$

for all $\Psi^{N} \in \operatorname{span}\left\{w_{1}, \ldots, w_{N}\right\} \subset H^{2}\left(\mathbb{R}^{2} ; \mathbb{R}^{2}\right)$ with $\operatorname{div} \Psi^{N}=0$, and $\psi^{N} \in$ $\operatorname{span}\left\{g_{1}, \ldots, g_{N}\right\} \subset H^{1}\left(\mathbb{R}^{2} ; \mathbb{R}\right)$. And $\mu^{n, N}$ is the classical solution to the transport equation

$$
\partial_{t} \mu^{n, N}+v^{n, N} \cdot \nabla \mu^{n, N}=0,\left.\quad \mu^{n, N}\right|_{t=0}=\phi_{n} * \mu_{0}
$$

The local in time existence for the system follows from the standard theory of ODEs, and in order to pass to the limit with $N$ we need just the a priori estimate in suitable energy norms independent of $N$, which of course depends on $T$ but never blows up for any finite $T$.

Note that the condition $\operatorname{div} v^{n, N}=0$ leads to the following bound uniformly in $N$ :

$$
\mu^{n, N} \in L^{\infty}\left(0, T ; L^{2}\left(\mathbb{R}^{2}\right)\right), \text { indeed } \mu^{n, N} \in L^{\infty}\left(0, T ; L^{1} \cap L^{\infty}\left(\mathbb{R}^{2}\right)\right)
$$

since by construction $\left\|\left.\mu^{n}\right|_{t=0}\right\|_{L^{1}\left(\mathbb{R}^{2}\right)} \leq C_{0}\left\|\mu_{0}\right\|_{\mathcal{M}\left(\mathbb{R}^{2}\right)}$ (uniformly in $n$ ). Hence testing the first equation by $\theta^{n, N}$ in (3.23) we get

$$
\theta^{n, N} \in L^{\infty}\left(0, T ; L^{2}\left(\mathbb{R}^{2}\right)\right) \cap L^{2}\left(0, T ; H^{1}\left(\mathbb{R}^{2}\right)\right), \text { uniformly in } N
$$

Then testing the second equation in (3.23) by $\Delta v^{n, N}$, and using the structure of the two dimensional Navier-Stokes equations we get

$$
v^{n, N} \in L^{\infty}\left(0, T ; H^{1}\left(\mathbb{R}^{2}\right)\right) \cap L^{2}\left(0, T ; H^{2}\left(\mathbb{R}^{2}\right)\right) \cap H^{1}\left(0, T ; L^{2}\left(\mathbb{R}^{2}\right)\right), \quad \text { uniformly in } N \text {. }
$$

The above information guarantees strong convergence of $\left(\mu^{n, N}, \theta^{n, N}, v^{n, N}\right)$ locally in space as $N \rightarrow \infty$. Hence there is no problem to pass to the limit $N \rightarrow \infty$ and we 
get the solution to the system (1.3) with initial data given by (3.21), that is:

$$
\begin{cases}\partial_{t} \mu^{n}+v^{n} \cdot \nabla \mu^{n}=0, & \text { in } \mathbb{R}^{2} \times(0, T], \\ \partial_{t} \theta^{n}+v^{n} \cdot \nabla \theta^{n}-\Delta \theta^{n}=\mu^{n}, & \text { in } \mathbb{R}^{2} \times(0, T], \\ \partial_{t} v^{n}+v^{n} \cdot \nabla v^{n}-\Delta v^{n}+\nabla p^{n}=\theta^{n}, & \text { in } \mathbb{R}^{2} \times(0, T], \\ \operatorname{div} v^{n}=0, & \text { in } \mathbb{R}^{2} \times(0, T], \\ \left.\mu^{n}\right|_{t=0},\left.\quad \theta^{n}\right|_{t=0},\left.\quad v^{n}\right|_{t=0} \in \mathcal{S}\left(\mathbb{R}^{2}\right) . & \end{cases}
$$

Using the standard bootstap method (here we use just the simple structure of quasilinear systems) we conclude that $\mu^{n}, \theta^{n}, v^{n}$ are smooth. Moreover we obtain that for every $n \in \mathbb{N}^{+}$and for any $1<q, p<\infty$,

$$
\begin{aligned}
& \mu^{n} \in L^{\infty}\left(0, T ; L^{1} \cap L^{\infty}\left(\mathbb{R}^{2}\right)\right), \\
& \theta^{n} \in L^{q}\left(0, T ; W^{2, p}\right) \cap W^{1, q}\left(0, T ; L^{p}\left(\mathbb{R}^{2}\right)\right), \\
& v^{n} \in L^{q}\left(0, T ; W^{4, p}\left(\mathbb{R}^{2}\right) \cap W^{2, q}\left(0, T ; L^{p}\left(\mathbb{R}^{2}\right)\right) .\right.
\end{aligned}
$$

To conclude (3.29) we note that thanks to (3.26) and (3.27), $v^{n} \cdot \nabla \theta^{n}$ belongs to $L^{2}\left(0, T ; L^{\bar{p}}\left(\mathbb{R}^{2}\right)\right)$ and $v^{n} \cdot \nabla v^{n}$ belong to $L^{2}\left(0, T ; W^{1, \bar{p}}\left(\mathbb{R}^{2}\right)\right)$ for every $\bar{p} \in[1,2)$; by the bootstrap method, one can improve the regularity to get that these products are in $L^{q}\left(0, T ; L^{\bar{p}}\left(\mathbb{R}^{2}\right)\right)$ and $L^{q}\left(0, T ; W^{1, \bar{p}}\left(\mathbb{R}^{2}\right)\right)$ for any $q \in(1, \infty)$, respectively; moreover, an another iteration leads to that for any $p>1, v^{n} \cdot \nabla \theta^{n} \in L^{q}\left(0, T ; L^{p}\left(\mathbb{R}^{2}\right)\right)$, $v^{n} \cdot \nabla v^{n} \in L^{q}\left(0, T ; W^{1, p}\left(\mathbb{R}^{2}\right)\right)$, and then applying the standard maximal regularity estimates in $L^{q}\left(0, T ; L^{p}\left(\mathbb{R}^{2}\right)\right)$ and $L^{q}\left(0, T ; W^{2, p}\left(\mathbb{R}^{2}\right)\right)$ for the heat and Stokes system, we get (3.29). Due to finiteness of $T$ factor $q$ can be decreased to anyone greater than one.

Sobolev embedding implies that $v^{n} \in L^{2}\left(0, T ; H^{4}\left(\mathbb{R}^{2}\right)\right) \cap L^{1}\left(0, T ; C^{3, \alpha}\left(\mathbb{R}^{2}\right)\right)$, $\alpha \in(0,1)$, and due to $\left.\mu^{n}\right|_{t=0} \in \mathcal{S}\left(\mathbb{R}^{2}\right)$, we moreover get $\mu^{n} \in L^{\infty}\left(0, T ; H^{4}\left(\mathbb{R}^{2}\right)\right) \cap$ $W^{1, \infty}\left(0, T ; H^{3}\left(\mathbb{R}^{2}\right)\right)$. Energy estimates and iteration ensure that the approximative sequence $\left(\mu^{n}, \theta^{n}, v^{n}\right)$ has sufficient smoothness so that the regularity assumptions in Propositions 3.1 - 3.3 are fulfilled. Hence we have the following uniform-in- $n$ estimates

$$
\left\|\mu^{n}\right\|_{L^{\infty}\left(0, T ; \mathcal{M}\left(\mathbb{R}^{2}\right)\right)} \leq\left\|\mu_{0}\right\|_{\mathcal{M}\left(\mathbb{R}^{2}\right)}, \quad \mu^{n} \geq 0, \quad \text { and } \quad \operatorname{supp} \mu^{n} \subset B_{R_{0}+C}(0) .
$$

and

$\left\|\theta^{n}\right\|_{L_{T}^{\infty}\left(L^{1} \cap B_{\frac{2}{2-\sigma}, \infty}^{2-\sigma}\right)}+\left\|v^{n}\right\|_{L_{T}^{\infty}\left(H^{1} \cap W^{1, \infty}\right)}+\left\|\left(\partial_{t} v^{n}, \nabla p^{n}, \nabla^{2} v^{n}\right)\right\|_{L_{T}^{\infty}\left(B_{\frac{2}{2-\sigma}, \infty}^{2-\sigma}\right)} \leq C$

where $C$ is depending on $T$ and norms of initial data $\left(\mu_{0}, \theta_{0}, v_{0}\right)$ but independent of $n \in \mathbb{N}^{+}$. Note that (3.29) with the trace theorem implies regularity needed for (3.31), 
since

$$
L^{q}\left(0, T ; W^{2, p}\right) \cap W^{1, q}\left(0, T ; L^{p}\left(\mathbb{R}^{2}\right)\right) \subset L^{\infty}\left(0, T ; B_{\frac{2}{2-\sigma}, \infty}^{2-\sigma}\left(\mathbb{R}^{2}\right)\right),
$$

taking $p=2 /(2-\sigma)$ and $q=2 / \sigma$.

Now we analyze a possible limit of the sequence as $n \rightarrow \infty$. For $v^{n}$ and $\theta^{n}$, due to (3.31), and based on the standard compactness argument for the Besov/Soblev spaces, we find a subsequence with strong (point-wise) convergence to some functions $v$ and $\theta$, more precisely, one has that for every $\varphi \in \mathcal{D}\left(\mathbb{R}^{2}\right)$,

$$
\begin{aligned}
& \varphi v^{n} \rightarrow \varphi v, \quad \text { in } L^{\infty}\left(0, T ; L^{2} \cap W^{1, \infty}\left(\mathbb{R}^{2}\right)\right), \\
& \varphi \theta^{n} \rightarrow \varphi \theta, \quad \text { in } L^{\infty}\left(0, T ; L^{2}\left(\mathbb{R}^{2}\right)\right) .
\end{aligned}
$$

For $\mu^{n}$, we view it as a mapping from $[0, T]$ to the metric space $\left(\mathcal{M}_{+}, d\right)$, and by arguing as a standard procedure in the transport theory (e.g. see [25]), we show that $\mu^{n}$ has a strong convergence through using the Arzela-Ascoli theorem. The uniform boundedness and relative compactness of $\mu^{n}(t)$ are followed from (3.30) and Proposition 2.3, and for the equicontinuity property of $\mu^{n}(t)$, we observe that for every $s_{1}, s_{2} \in[0, T]$ and every $\pi \in W^{1, \infty}\left(\mathbb{R}^{2}\right)$,

$$
\left|\int_{\mathbb{R}^{2}}\left(\mu^{n}\left(s_{2}\right)-\mu^{n}\left(s_{1}\right)\right) \pi \mathrm{d} x\right|=\left|\int_{s_{1}}^{s_{2}} \int_{\mathbb{R}^{2}} v^{n} \mu^{n} \nabla \pi \mathrm{d} x \mathrm{~d} t\right| \leq C \operatorname{Lip}(\pi)\left\|\mu_{0}\right\|_{\mathcal{M}}\left|s_{2}-s_{1}\right|,
$$

so that

$$
d\left(\mu^{n}\left(s_{2}\right), \mu^{n}\left(s_{1}\right)\right) \leq C\left|s_{2}-s_{1}\right| .
$$

Thus the assumptions of Arzela-Ascoli theorem are satisfied and there exists $\mu \in$ $L^{\infty}\left(0, T ; \mathcal{M}_{+}\right)$such that, up to a subsequence,

$$
\mu^{n}(t) \rightarrow \mu(t) \text { in } d \text {-topology uniformly in time. }
$$

The information (3.30) also ensures $\mu \in L^{\infty}\left(0, T ; \mathcal{M}_{+}\left(\mathbb{R}^{2}\right)\right)$ and $\operatorname{supp} \mu \subset$ $B_{R_{0}+C}(0)$. In addition, in view of Definition 2.1, the bound (3.34) and strong convergence of the velocity in $L^{\infty}\left(0, T ; W_{\text {loc }}^{1, \infty}\left(\mathbb{R}^{2}\right)\right)$ guarantee that as $n \rightarrow \infty$ we have

$$
v^{n} \mu^{n} \rightarrow v \mu \quad \text { in } \mathcal{D}^{\prime}\left(\mathbb{R}^{2} \times[0, T]\right)
$$

We thus have the existence. 


\subsection{Uniqueness}

Consider two solutions $\left(\mu_{1}, \theta_{1}, v_{1}, p_{1}\right)$ and $\left(\mu_{2}, \theta_{2}, v_{2}, p_{2}\right)$ to the Boussinesq type system (1.3) starting from the same initial data $\left(\mu_{0}, \theta_{0}, v_{0}\right)$ as stated in Theorem 1.1. According to Proposition 3.3, we know that for $i=1,2$ and for any $T>0$,

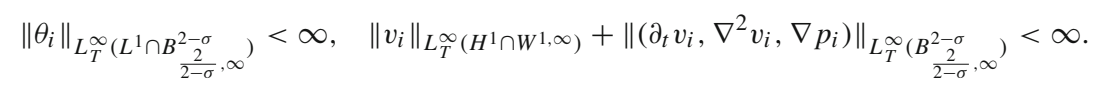

Denoting by $\bar{v}_{i}(t, y)=v_{i}\left(t, X_{v_{i}}(t, y)\right)$ with $X_{v_{i}}(t, y)$ the particle-trajectory generated by $v_{i}$ (see (2.15)), we immediately obtain that

$$
\left\|\nabla_{y} \bar{v}_{i}\right\|_{L_{T}^{\infty}\left(L^{\infty}\right)} \leq\left\|\nabla_{x} v_{i}\right\|_{L_{T}^{\infty}\left(L^{\infty}\right)}\left\|\nabla_{y} X_{v_{i}}\right\|_{L_{T}^{\infty}\left(L^{\infty}\right)} \leq\left\|\nabla v_{i}\right\|_{L_{T}^{\infty}\left(L^{\infty}\right)} e^{\int_{0}^{T}\left\|\nabla v_{i}(t)\right\|_{L^{\infty}} \mathrm{d} t}<\infty,
$$

and moreover by letting $T^{\prime}>0$ be small enough, we can have

$$
\int_{0}^{T^{\prime}}\left\|\nabla_{x} v_{i}(t)\right\|_{L^{\infty}} \mathrm{d} t \leq \frac{1}{2}, \quad \text { and } \quad \int_{0}^{T^{\prime}}\left\|\nabla_{y} \bar{v}_{i}(t)\right\|_{L^{\infty}} \mathrm{d} t \leq T^{\prime}\left\|\nabla_{y} \bar{v}_{i}\right\|_{L_{T}^{\infty}\left(L^{\infty}\right)} \leq \frac{1}{2} .
$$

As a consequence of (3.36) and (3.37), we also infer that

$$
\left\|\bar{\theta}_{i}\right\|_{L_{T}^{\infty}\left(L^{4}\right)}+\left\|\nabla_{y}^{2} \bar{v}_{i}\right\|_{L_{T}^{\infty}\left(L^{4}\right)}+\left\|\partial_{t} \bar{v}_{i}\right\|_{L_{T}^{\infty}\left(L^{4}\right)}+\left\|\nabla_{y} \bar{p}_{i}\right\|_{L_{T}^{\infty}\left(L^{4}\right)}<\infty .
$$

To keep the simple form of estimates we restrict ourselves to the case $\frac{2}{2-\sigma} \leq 4$ (i.e. $\left.\sigma \in\left(0, \frac{3}{2}\right]\right)$ The continuous embedding implies

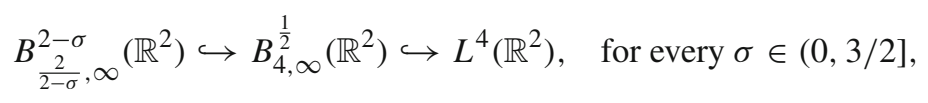

For general case we refer to Remark 1.2. Next, we find

$$
\begin{aligned}
\left\|\bar{\theta}_{i}\right\|_{L_{T}^{\infty}\left(L^{4}\right)} & \leq\left\|\theta_{i}\right\|_{L_{T}^{\infty}\left(L^{4}\right)} \leq C\left\|\theta_{i}\right\|_{L_{T}^{\infty}\left(B_{\frac{2}{2-\sigma}, \infty}^{2-\sigma}\right)}<\infty, \\
\left\|\left(\partial_{t} v_{i}, \nabla^{2} v_{i}, \nabla p_{i}\right)\right\|_{L_{T}^{\infty}\left(L^{4}\right)} & \leq C\left\|\left(\partial_{t} v_{i}, \nabla^{2} v_{i}, \nabla p_{i}\right)\right\|_{L_{T}^{\infty}\left(B_{\frac{2}{2-\sigma}, \infty}^{2-\sigma}\right)}<\infty,
\end{aligned}
$$

and thanks to estimates (2.18), (2.21),

$$
\begin{aligned}
\left\|\nabla_{y}^{2} \bar{v}_{i}\right\|_{L_{T}^{\infty}\left(L^{4}\right)} \leq & \left.C\left\|\nabla X_{v_{i}}\right\|_{L_{T}^{\infty}\left(L^{\infty}\right)}^{2}\left\|\nabla^{2} v_{i}\right\|_{L_{T}^{\infty}\left(B_{\frac{2}{2-\sigma}, \infty}^{2-\sigma}\right)}{ }\right) \\
& +\left\|\nabla v_{i}\right\|_{L_{T}^{\infty}\left(L^{\infty}\right)}\left\|\nabla^{2} X_{v_{i}}\right\|_{L_{T}^{\infty}\left(L^{4}\right)}<\infty
\end{aligned}
$$


and

$$
\begin{aligned}
\left\|\partial_{t} \bar{v}_{i}\right\|_{L_{T}^{\infty}\left(L^{4}\right)} & \leq C\left\|\partial_{t} v_{i}\right\|_{L_{T}^{\infty}\left(B_{\frac{2}{2-\sigma}, \infty}^{2-\sigma}\right)}+C\left\|\nabla v_{i}\right\|_{L_{T}^{\infty}\left(B_{\frac{2}{2-\sigma}, \infty}^{2-\sigma}\right)}\left\|v_{i}\right\|_{L_{T}^{\infty}\left(L^{\infty}\right)}<\infty, \\
\left\|\nabla \bar{p}_{i}\right\|_{L_{T}^{\infty}\left(L^{4}\right)} & \leq\left\|\nabla_{x} p_{i}\left(t, X_{v_{i}}\right)\right\|_{L_{T}^{\infty}\left(L^{4}\right)}\left\|\nabla X_{v_{i}}\right\|_{L_{T}^{\infty}\left(L^{\infty}\right)} \\
& \leq C\left\|\nabla p_{i}\right\|_{L_{T}^{\infty}\left(B_{\frac{2}{2-\sigma}, \infty}^{2-\sigma}\right)}\left\|\nabla X_{v_{i}}\right\|_{L_{T}^{\infty}\left(L^{\infty}\right)}<\infty .
\end{aligned}
$$

By adopting the notations introduced in Sect. 2.3 and using (2.26), the system of $\left(\mu_{i}, \theta_{i}, v_{i}, p_{i}\right)(i=1,2)$ in the Lagrangian coordinates is written as

$$
\left\{\begin{array}{l}
\partial_{t} \bar{\theta}_{i}-\Delta \bar{\theta}_{i}=\mu_{0}+N_{1}\left(A_{v_{i}}, \nabla \bar{\theta}_{i}\right), \\
\partial_{t} \bar{v}_{i}-\Delta \bar{v}_{i}+\nabla_{y} \bar{p}_{i}=\bar{\theta}_{i} e_{2}+N_{2}\left(A_{v_{i}}, \nabla \bar{v}_{i}\right)+N_{3}\left(A_{v_{i}}, \nabla \bar{p}_{i}\right), \\
\operatorname{div} \bar{v}_{i}=\operatorname{div}\left(\left(\operatorname{Id}-A_{v_{i}}\right) \bar{v}_{i}\right)=\left(\operatorname{Id}-A_{v_{i}}^{\mathrm{T}}\right): \nabla \bar{v}_{i}, \\
\left.\bar{\theta}_{i}\right|_{t=0}=\theta_{0},\left.\quad \bar{v}_{i}\right|_{t=0}=v_{0},
\end{array}\right.
$$

where nonlinear terms $N_{1}, N_{2}, N_{3}$ are given by (2.27)-(2.28). The choice of the Lagrangian coordinates setting removes the problem with uniqueness for measure force $\mu$. They are now given explicitly as follows

$$
\mu_{1}\left(t, X_{v_{1}}(t, y)\right)=\mu_{2}\left(t, X_{v_{2}}(t, y)\right)=\mu_{0}(y) \text {. }
$$

The difference equations of $\delta \bar{\theta}:=\bar{\theta}_{1}-\bar{\theta}_{2}, \delta \bar{v}:=\bar{v}_{1}-\bar{v}_{2}$ and $\delta \bar{p}:=\bar{p}_{1}-\bar{p}_{2}$ read as follows

$$
\left\{\begin{array}{l}
\partial_{t} \delta \bar{\theta}-\Delta \delta \bar{\theta}=\delta N_{1}, \\
\partial_{t} \delta \bar{v}-\Delta \delta \bar{v}+\nabla \delta \bar{p}=(\delta \bar{\theta}) e_{2}+\delta N_{2}+\delta N_{3} \\
\operatorname{div} \delta \bar{v}=\operatorname{div}\left(\delta N_{4}\right) \\
\left.\delta \bar{\theta}\right|_{t=0}=0,\left.\quad \delta \bar{v}\right|_{t=0}=0
\end{array}\right.
$$

with

$$
\begin{aligned}
& \delta N_{1}:=\operatorname{div}\left(\left(A_{v_{1}} A_{v_{1}}^{\mathrm{T}}-A_{v_{2}} A_{v_{2}}^{\mathrm{T}}\right) \nabla \bar{\theta}_{2}\right)-\operatorname{div}\left(\left(\operatorname{Id}-A_{v_{1}} A_{v_{1}}^{\mathrm{T}}\right) \nabla \delta \bar{\theta}\right), \\
& \delta N_{2}:=\operatorname{div}\left(\left(A_{v_{1}} A_{v_{1}}^{\mathrm{T}}-A_{v_{2}} A_{v_{2}}^{\mathrm{T}}\right) \nabla \bar{v}_{2}\right)-\operatorname{div}\left(\left(\operatorname{Id}-A_{v_{1}} A_{v_{1}}^{\mathrm{T}}\right) \nabla \delta \bar{v}\right), \\
& \delta N_{3}:=-\left(A_{v_{1}}^{\mathrm{T}}-A_{v_{2}}^{\mathrm{T}}\right) \nabla \bar{p}_{2}+\left(\operatorname{Id}-A_{v_{1}}^{\mathrm{T}}\right) \nabla \delta \bar{p}, \\
& \delta N_{4}:=\left(A_{v_{1}}-A_{v_{2}}\right) \bar{v}_{2}+\left(\mathrm{Id}-A_{v_{1}}\right) \delta \bar{v} .
\end{aligned}
$$

The target here is to show uniqueness in the following functional spaces: $\delta \bar{\theta} \in L^{2}\left(0, T ; L^{2}\left(\mathbb{R}^{2}\right)\right), \quad$ and $\quad \nabla \delta \bar{v} \in L^{\infty}\left(0, T ; L^{2}\left(\mathbb{R}^{2}\right)\right) \cap L^{2}\left(0, T ; \dot{H}^{1}\left(\mathbb{R}^{2}\right)\right)$. 
In order to perform the $L_{T}^{2}\left(L^{2}\right)$-estimate of $\delta \bar{\theta}$, we introduce a function $w$ which solves the backward heat equation

$$
\partial_{t} w+\Delta w=\delta \bar{\theta}, \text { and }\left.w\right|_{t=T^{\prime}}=0,
$$

with $T^{\prime} \in(0, T]$ being any given. Standard energy estimates yield the following result (for the proof see the appendix).

Lemma 3.4 Let $\delta \bar{\theta} \in L^{2}\left(0, T^{\prime} ; L^{2}\left(\mathbb{R}^{2}\right)\right)$, then there exists a unique weak solution $w \in L^{\infty}\left(0, T^{\prime} ; H^{1}\right) \cap L^{2}\left(0, T ; H^{2}\right)$ which satisfies

$$
\sup _{t \in\left[0, T^{\prime}\right]}\|\nabla w\|_{L^{2}\left(\mathbb{R}^{2}\right)}^{2}+\int_{0}^{T^{\prime}}\left\|\nabla^{2} w, \partial_{t} w\right\|_{L^{2}}^{2} \mathrm{~d} t+\|\nabla w\|_{L^{4}\left(0, T^{\prime} ; L^{4}\right)}^{2} \leq C_{0}\|\delta \bar{\theta}\|_{L^{2}\left(0, T^{\prime} ; L^{2}\right)}^{2},
$$

with $C_{0}>0$ a universal constant independent of $T^{\prime}$.

Now we take the space-time scalar product of the first equation of (3.43) with $w$, and observing that (which can be justified by integration by parts and an approximation procedure)

$$
\int_{0}^{T^{\prime}} \int_{\mathbb{R}^{2}}\left(\partial_{t} \delta \bar{\theta}-\Delta \delta \bar{\theta}\right) w \mathrm{~d} x \mathrm{~d} t=\int_{0}^{T^{\prime}} \int_{\mathbb{R}^{2}} \delta \bar{\theta}\left(-\partial_{t} w-\Delta w\right) \mathrm{d} x \mathrm{~d} t,
$$

we find that

$$
\begin{aligned}
\|\delta \bar{\theta}\|_{L^{2}\left(0, T^{\prime} ; L^{2}\left(\mathbb{R}^{2}\right)\right) \leq} \leq\left|\int_{0}^{T^{\prime}} \int_{\mathbb{R}^{2}}\left(\delta N_{1}\right) w \mathrm{~d} x \mathrm{~d} t\right| \\
\leq\left|\int_{0}^{T^{\prime}} \int_{\mathbb{R}^{2}}\left(\left(A_{v_{1}} A_{v_{1}}^{\mathrm{T}}-A_{v_{2}} A_{v_{2}}^{\mathrm{T}}\right) \nabla \bar{\theta}_{2}\right) \cdot \nabla w \mathrm{~d} x \mathrm{~d} t\right| \\
+\left|\int_{0}^{T^{\prime}} \int_{\mathbb{R}^{2}}\left(\left(\mathrm{Id}-A_{v_{1}} A_{v_{1}}^{\mathrm{T}}\right) \nabla \delta \bar{\theta}\right) \cdot \nabla w \mathrm{~d} x \mathrm{~d} t\right| \\
\leq \\
+\left|\int_{0}^{T^{\prime}} \int_{\mathbb{R}^{2}}\left(\left(A_{v_{1}} A_{v_{1}}^{\mathrm{T}}-A_{v_{2}} A_{v_{2}}^{\mathrm{T}}\right) \bar{\theta}_{2}\right) \cdot \nabla^{2} w \mathrm{~d} x \mathrm{~d} t\right| \\
+\left|\int_{0}^{T^{\prime}} \int_{\mathbb{R}^{2}}\left(\nabla\left(A_{v_{1}} A_{v_{1}}^{\mathrm{T}}-A_{v_{2}} A_{v_{2}}^{\mathrm{T}}\right) \overline{\theta_{2}}\right) \cdot \nabla w \mathrm{~d} x \mathrm{~d} t\right| \\
+\left|\int_{0}^{T^{\prime}} \int_{\mathbb{R}^{2}}\left(\left(\operatorname{Id}-A_{v_{1}} A_{v_{1}}^{\mathrm{T}}\right) \delta \bar{\theta}\right) \cdot \nabla^{2} w \mathrm{~d} x \mathrm{~d} t\right| \\
+\left|\int_{0}^{T^{\prime}} \int_{\mathbb{R}^{2}}\left(\nabla\left(\operatorname{Id}-A_{v_{1}} A_{v_{1}}^{\mathrm{T}}\right) \delta \bar{\theta}\right) \cdot \nabla w \mathrm{~d} x \mathrm{~d} t\right| \\
:=
\end{aligned}
$$


For latter use, we recall an auxiliary lemma on some estimates of $A_{v}$ given by (2.24).

Lemma 3.5 Assume that $v_{1}, v_{2} \in L^{1}\left(0, T ; W^{1, \infty}\left(\mathbb{R}^{d}\right)\right)$ are two vector fields such that

$$
\int_{0}^{T}\left\|\nabla_{y} \bar{v}_{i}\right\|_{L^{\infty}} \mathrm{d} t \leq \frac{1}{2}, \quad \text { for } i=1,2
$$

Then we have that for every $p \in[1, \infty]$ and $i=1,2$,

$$
\begin{aligned}
\left\|A_{v_{i}}\right\|_{L_{T}^{\infty}\left(L^{\infty}\right)} & \leq 1 \\
\left\|\mathrm{Id}-A_{v_{i}}\right\|_{L_{T}^{\infty}\left(L^{\infty}\right)} & \leq 2\left\|\nabla \bar{v}_{i}\right\|_{L_{T}^{1}\left(L^{\infty}\right)}, \\
\left\|A_{v_{1}}-A_{v_{2}}\right\|_{L_{T}^{\infty}\left(L^{p}\right)} & \leq C_{0}\|\nabla \delta \bar{v}\|_{L_{T}^{1}\left(L^{p}\right)}, \\
\left\|\nabla A_{v_{i}}\right\|_{L_{T}^{\infty}\left(L^{p}\right)} & \leq C_{0}\left\|\nabla^{2} \bar{v}_{i}\right\|_{L_{T}^{1}\left(L^{p}\right)}, \\
\left\|\nabla A_{v_{1}}-\nabla A_{v_{2}}\right\|_{L_{T}^{\infty}\left(L^{2}\right)} & \leq C_{0}\left\|\nabla^{2} \delta \bar{v}\right\|_{L_{T}^{1}\left(L^{2}\right)}+C_{0}\|\nabla \delta \bar{v}\|_{L_{T}^{1}\left(L^{4}\right)}\left\|\nabla^{2} \bar{v}_{1}\right\|_{L_{T}^{1}\left(L^{4}\right)},
\end{aligned}
$$

$$
\left\|\partial_{t} A_{v_{i}}\right\|_{L_{T}^{\infty}\left(L^{p}\right)} \leq C_{0}\left\|\nabla \bar{v}_{i}\right\|_{L_{T}^{\infty}\left(L^{p}\right)},
$$

$$
\left\|\partial_{t} A_{v_{1}}-\partial_{t} A_{v_{2}}\right\|_{L_{T}^{\infty}\left(L^{2}\right)} \leq C_{0}\left(1+T\left\|\nabla \bar{v}_{1}\right\|_{L_{T}^{\infty}\left(L^{\infty}\right)}\right)\|\nabla \delta \bar{v}\|_{L_{T}^{\infty}\left(L^{2}\right)},
$$

where $C_{0}>0$ is an absolute constant.

By applying estimates (3.53), (3.55), (3.38), (3.50), and Young's inequality, we treat the term $\mathrm{I}_{1}$ as

$$
\begin{aligned}
\mathrm{I}_{1} \leq & T^{\prime \frac{1}{2}}\left\|A_{v_{1}}-A_{v_{2}}\right\|_{L_{T^{\prime}}^{\infty}\left(L^{4}\right)}\left\|\left(A_{v_{1}}, A_{v_{2}}\right)\right\|_{L_{T^{\prime}}^{\infty}\left(L^{\infty}\right)}\left\|\bar{\theta}_{2}\right\|_{L_{T^{\prime}}^{\infty}\left(L^{4}\right)}\left\|\nabla^{2} w\right\|_{L_{T^{\prime}}^{2}\left(L^{2}\right)} \\
\leq & C T^{\prime \frac{5}{4}}\|\nabla \delta \bar{v}\|_{L_{T^{\prime}}^{4}\left(L^{4}\right)}\left\|\bar{\theta}_{2}\right\|_{L_{T^{\prime}}^{\infty}\left(L^{4}\right)}\|\delta \bar{\theta}\|_{L_{T^{\prime}}^{2}\left(L^{2}\right)} \leq \frac{1}{8}\|\delta \bar{\theta}\|_{L_{T^{\prime}}^{2}\left(L^{2}\right)}^{2} \\
& +C T^{\prime \frac{5}{2}}\|\nabla \delta \bar{v}\|_{L_{T^{\prime}}^{4}\left(L^{4}\right)}^{2}\left\|\bar{\theta}_{2}\right\|_{L_{T^{\prime}}^{\infty}\left(L^{4}\right)}^{2} .
\end{aligned}
$$

For term $\mathrm{I}_{2}$, by virtue of (3.53)-(3.57) and (3.50), we get that

$$
\begin{aligned}
\mathrm{I}_{2} \leq & C T^{\prime \frac{3}{4}}\|\nabla w\|_{L_{T^{\prime}}^{4}\left(L^{4}\right)}\left\|\bar{\theta}_{2}\right\|_{L_{T^{\prime}}^{\infty}\left(L^{4}\right)}\left\|\nabla A_{v_{1}}-\nabla A_{v_{2}}\right\|_{L_{T^{\prime}}^{\infty}\left(L^{2}\right)}\left\|A_{v_{2}}\right\|_{L_{T^{\prime}}^{\infty}\left(L^{\infty}\right)} \\
& +C T^{\prime \frac{3}{4}}\|\nabla w\|_{L_{T^{\prime}}^{4}\left(L^{4}\right)}\left\|\bar{\theta}_{2}\right\|_{L_{T^{\prime}}^{\infty}\left(L^{4}\right)}\left\|A_{v_{1}}-A_{v_{2}}\right\|_{L_{T^{\prime}}^{\infty}\left(L^{4}\right)}\left\|\nabla A_{v_{1}}\right\|_{L_{T^{\prime}}^{\infty}\left(L^{4}\right)} \\
\leq & C T^{\prime \frac{3}{4}}\|\delta \bar{\theta}\|_{L_{T^{\prime}}^{2}\left(L^{2}\right)}\left\|\bar{\theta}_{2}\right\|_{L_{T^{\prime}}^{\infty}\left(L^{4}\right)}\left(T^{\prime \frac{1}{2}}\left\|\nabla^{2} \delta \bar{v}\right\|_{L_{T^{\prime}}^{2}\left(L^{2}\right)}\right. \\
& \left.+T^{\prime \frac{7}{4}}\|\nabla \delta \bar{v}\|_{L_{T^{\prime}}^{4}\left(L^{4}\right)}\left\|\nabla^{2} \bar{v}_{1}\right\|_{L_{T^{\prime}}^{\infty}\left(L^{4}\right)}\right) \\
\leq & \frac{1}{8}\|\delta \bar{\theta}\|_{L_{T^{\prime}\left(L^{2}\right)}^{2}}^{2}+C T^{\prime \frac{5}{2}}\left\|\bar{\theta}_{2}\right\|_{L_{T^{\prime}}^{\infty}\left(L^{4}\right)}^{2}\left(\left\|\nabla^{2} \delta \bar{v}\right\|_{L_{T^{\prime}}^{2}\left(L^{2}\right)}^{2}\right. \\
& \left.+T^{\prime \frac{5}{2}}\left\|\nabla^{2} \bar{v}_{1}\right\|_{L_{T^{\prime}}^{\infty}\left(L^{4}\right)}^{2}\|\nabla \delta \bar{v}\|_{L_{T^{\prime}}^{4}\left(L^{4}\right)}^{2}\right) .
\end{aligned}
$$


For term $\mathrm{I}_{3}$, the estimates (3.53), (3.54) and (3.50) directly guarantee that

$$
\begin{aligned}
\mathrm{I}_{3} & \leq 2\left\|\mathrm{Id}-A_{v_{1}}\right\|_{L_{T^{\prime}}^{\infty}\left(L^{\infty}\right)}\left\|A_{v_{1}}\right\|_{L_{T^{\prime}}^{\infty}\left(L^{\infty}\right)}\|\delta \bar{\theta}\|_{L_{T^{\prime}}^{2}\left(L^{2}\right)}\left\|\nabla^{2} w\right\|_{L_{T^{\prime}}^{2}\left(L^{2}\right)} \\
& \leq 4 T^{\prime}\left\|\nabla \bar{v}_{1}\right\|_{L_{T^{\prime}}^{\infty}\left(L^{\infty}\right)}\|\delta \bar{\theta}\|_{L_{T^{\prime}}^{2}\left(L^{2}\right)^{2}}^{2} .
\end{aligned}
$$

For term $\mathrm{I}_{4}$, thanks to (3.56), (3.50) and Young's inequality again, we deduce

$$
\begin{aligned}
\mathrm{I}_{4} & \leq 2\left\|\nabla A_{v_{1}}\right\|_{L_{T^{\prime}}^{\infty}\left(L^{4}\right)}\left\|A_{v_{1}}\right\|_{L_{T^{\prime}}^{\infty}\left(L^{\infty}\right)}\|\delta \bar{\theta}\|_{L_{T^{\prime}}^{2}\left(L^{2}\right)}\|\nabla w\|_{L_{T^{\prime}}^{2}\left(L^{4}\right)} \\
& \leq C T^{\prime \frac{5}{4}}\left\|\nabla^{2} \bar{v}_{1}\right\|_{L_{T^{\prime}}^{\infty}\left(L^{4}\right)}\|\delta \bar{\theta}\|_{L_{T^{\prime}}^{2}\left(L^{2}\right)^{2}} .
\end{aligned}
$$

Gathering (3.51) and the above estimates on $\mathrm{I}_{1}-\mathrm{I}_{4}$, and also letting $T^{\prime}>0$ be sufficiently small so that

$$
T^{\prime}\left\|\nabla \bar{v}_{1}\right\|_{L_{T^{\prime}}^{\infty}\left(L^{\infty}\right)} \leq \frac{1}{32}, \quad \text { and } \quad C T^{\prime \frac{5}{4}}\left\|\nabla^{2} \bar{v}_{1}\right\|_{L_{T}^{\infty}\left(L^{4}\right)} \leq \frac{1}{8}
$$

we get

$$
\|\delta \bar{\theta}\|_{L_{T^{\prime}}^{2}\left(L^{2}\right)}^{2} \leq C T^{\prime \frac{5}{2}}\left\|\bar{\theta}_{2}\right\|_{L_{T^{\prime}}^{\infty}\left(L^{4}\right)}^{2}\left(\left\|\nabla^{2} \delta \bar{v}\right\|_{L_{T^{\prime}}^{2}\left(L^{2}\right)}^{2}+\|\nabla \delta \bar{v}\|_{L_{T^{\prime}}^{4}\left(L^{4}\right)}^{2}\right) .
$$

Next we turn to the estimation of $\delta \bar{v}$. According to Lemma 2.7 and the interpolation inequality

$$
\|\nabla f\|_{L_{T^{\prime}}^{4}\left(L^{4}\left(\mathbb{R}^{2}\right)\right)} \leq C_{0}\|\nabla f\|_{L_{T^{\prime}}^{\infty}\left(L^{2}\left(\mathbb{R}^{2}\right)\right)}^{1 / 2}\left\|\nabla^{2} f\right\|_{L_{T^{\prime}}^{2}\left(L^{2}\left(\mathbb{R}^{2}\right)\right)}^{1 / 2},
$$

we have

$$
\begin{aligned}
& \|\nabla \delta \bar{v}\|_{L_{T^{\prime}}^{\infty}\left(L^{2}\right)}+\left\|\left(\partial_{t} \delta \bar{v}, \nabla^{2} \delta \bar{v}, \nabla \delta \bar{p}\right)\right\|_{L_{T^{\prime}}^{2}\left(L^{2}\right)}+\|\nabla \delta \bar{v}\|_{L_{T^{\prime}}^{4}\left(L^{4}\right)} \\
& \leq C\|\delta \bar{\theta}\|_{L_{T^{\prime}}^{2}\left(L^{2}\right)}+C\left\|\delta N_{2}\right\|_{L_{T^{\prime}}^{2}\left(L^{2}\right)}+C\left\|\delta N_{3}\right\|_{L_{T^{\prime}}^{2}\left(L^{2}\right)} \\
& \left.\quad+C\left\|\partial_{t}\left(\delta N_{4}\right)\right\|_{L_{T^{\prime}}^{2}\left(L^{2}\right)}+C\left\|\nabla \operatorname{div}\left(\delta N_{4}\right)\right\|_{L_{T^{\prime}}^{2}\left(L^{2}\right)}:=\mathrm{II}_{1}+\mathrm{II}_{2}+\mathrm{II}_{3}+\mathrm{II}_{4}+3 \mathrm{II}_{3},\right)
\end{aligned}
$$

where $\delta N_{2}, \delta N_{3}, \delta N_{4}$ are given by (3.45)-(3.47). For $\mathrm{II}_{2}$, similarly as estimating of $\mathrm{I}_{2}$, from (3.38) and (3.53)-(3.57), we have

$$
\begin{aligned}
\mathrm{II}_{2} \leq & C\left\|\nabla\left(A_{v_{1}} A_{v_{1}}^{\mathrm{T}}-A_{v_{2}} A_{v_{2}}^{\mathrm{T}}\right) \cdot \nabla \bar{v}_{2}\right\|_{L_{T^{\prime}}^{2}\left(L^{2}\right)}+C\left\|\left(A_{v_{1}} A_{v_{1}}^{\mathrm{T}}-A_{v_{2}} A_{v_{2}}^{\mathrm{T}}\right) \cdot \nabla^{2} \bar{v}_{2}\right\|_{L_{T^{\prime}}^{2}\left(L^{2}\right)} \\
& +C\left\|\left(\mathrm{Id}-A_{v_{1}} A_{v_{1}}^{\mathrm{T}}\right) \cdot \nabla^{2} \delta \bar{v}\right\|_{L_{T^{\prime}}^{2}\left(L^{2}\right)}+C\left\|\nabla\left(A_{v_{1}} A_{v_{1}}^{\mathrm{T}}\right) \nabla \delta \bar{v}\right\|_{L_{T^{\prime}}^{2}\left(L^{2}\right)} \\
\leq & C T^{\prime \frac{1}{2}}\left\|\nabla \bar{v}_{2}\right\|_{L_{T^{\prime}}^{\infty}\left(L^{\infty}\right)}\left(\left\|\nabla^{2} \delta \bar{v}\right\|_{L_{T^{\prime}}^{1}\left(L^{2}\right)}+\|\nabla \delta \bar{v}\|_{L_{T^{\prime}}^{1}\left(L^{4}\right)}\left\|\nabla^{2} \bar{v}_{1}\right\|_{L_{T^{\prime}}^{1}\left(L^{4}\right)}\right) \\
& +C\|\nabla \delta \bar{v}\|_{L_{T^{\prime}}^{1}\left(L^{4}\right)}\left\|\nabla^{2} \bar{v}_{2}\right\|_{L_{T^{\prime}}^{2}\left(L^{4}\right)}+C\left\|\nabla \bar{v}_{1}\right\|_{L_{T^{\prime}}^{1}\left(L^{\infty}\right)}\left\|\nabla^{2} \delta \bar{v}\right\|_{L_{T^{\prime}}^{2}\left(L^{2}\right)} \\
& +C\left\|\nabla^{2} \bar{v}_{1}\right\|_{L_{T^{\prime}}^{1}\left(L^{4}\right)}\|\nabla \delta \bar{v}\|_{L_{T^{\prime}}^{2}\left(L^{4}\right)}
\end{aligned}
$$




$$
\leq C T^{\prime}\left\|\left(\nabla \bar{v}_{1}, \nabla \bar{v}_{2}\right)\right\|_{L_{T^{\prime}}^{\infty}\left(L^{\infty}\right)}\left\|\nabla^{2} \delta \bar{v}\right\|_{L_{T^{\prime}}^{2}\left(L^{2}\right)}+C T^{\prime \frac{5}{4}}\left\|\left(\nabla^{2} \bar{v}_{1}, \nabla^{2} \bar{v}_{2}\right)\right\|_{L_{T^{\prime}}^{\infty}\left(L^{4}\right)}\|\nabla \delta \bar{v}\|_{L_{T^{\prime}}^{4}\left(L^{4}\right)} .
$$

For $\mathrm{II}_{3},(3.53)-(3.56)$ and Hölder's inequality lead to that

$$
\begin{aligned}
\mathrm{II}_{3} & \leq C\left\|\left(A_{v_{1}}^{\mathrm{T}}-A_{v_{2}}^{\mathrm{T}}\right) \nabla \bar{p}_{2}\right\|_{L_{T^{\prime}}^{2}\left(L^{2}\right)}+C\left\|\left(\mathrm{Id}-A_{v_{1}}^{\mathrm{T}}\right) \nabla \delta \bar{p}\right\|_{L_{T^{\prime}}^{2}\left(L^{2}\right)} \\
& \leq C T^{\prime \frac{1}{2}}\left\|A_{v_{1}}-A_{v_{2}}\right\|_{L_{T^{\prime}}^{\infty}\left(L^{4}\right)}\left\|\nabla \bar{p}_{2}\right\|_{L_{T^{\prime}}^{\infty}\left(L^{4}\right)}+C\left\|\mathrm{Id}-A_{v_{1}}\right\|_{L_{T^{\prime}}^{\infty}\left(L^{\infty}\right)}\|\nabla \delta \bar{p}\|_{L_{T^{\prime}}^{2}\left(L^{2}\right)} \\
& \leq C T^{\prime \frac{5}{4}}\|\nabla \delta \bar{v}\|_{L_{T^{\prime}}^{4}\left(L^{4}\right)}\left\|\nabla \bar{p}_{2}\right\|_{L_{T^{\prime}}^{\infty}\left(L^{4}\right)}+C T^{\prime}\left\|\nabla \bar{v}_{1}\right\|_{L_{T^{\prime}}^{\infty}\left(L^{\infty}\right)}\|\nabla \delta \bar{p}\|_{L_{T^{\prime}}^{2}\left(L^{2}\right)^{\prime}} .
\end{aligned}
$$

For $\mathrm{II}_{4}$, through applying estimates (3.38), (3.55), (3.58), (3.59), it follows that

$$
\begin{aligned}
\mathrm{II}_{4} \leq & C\left\|\partial_{t}\left(\left(A_{v_{1}}-A_{2}\right) \bar{v}_{2}\right)\right\|_{L_{T^{\prime}}^{2}\left(L^{2}\right)}+C\left\|\partial_{t}\left(\left(\mathrm{Id}-A_{v_{1}}\right) \delta \bar{v}\right)\right\|_{L_{T^{\prime}}^{2}\left(L^{2}\right)} \\
\leq & C T^{\prime \frac{1}{2}}\left\|\partial_{t}\left(A_{v_{1}}-A_{v_{2}}\right)\right\|_{L_{T^{\prime}}^{\infty}\left(L^{2}\right)}\left\|\bar{v}_{2}\right\|_{L_{T^{\prime}}^{\infty}\left(L^{\infty}\right)} \\
& +C T^{\prime \frac{1}{2}}\left\|A_{v_{1}}-A_{v_{2}}\right\|_{L_{T^{\prime}}^{\infty}\left(L^{4}\right)}\left\|\partial_{t} \bar{v}_{2}\right\|_{L_{T^{\prime}}^{\infty}\left(L^{4}\right)} \\
& +C T^{\prime \frac{1}{2}}\left\|\partial_{t} A_{v_{1}}\right\|_{L_{T^{\prime}}^{\infty}\left(L^{\infty}\right)}\|\delta \bar{v}\|_{L_{T^{\prime}}^{\infty}\left(L^{2}\right)}+C\left\|\mathrm{Id}-A_{v_{1}}\right\|_{L_{T^{\prime}}^{\infty}\left(L^{\infty}\right)}\left\|\partial_{t} \delta \bar{v}\right\|_{L_{T^{\prime}}^{2}\left(L^{2}\right)} \\
\leq & C T^{\prime \frac{1}{2}}\|\nabla \delta \bar{v}\|_{L_{T^{\prime}}^{\infty}\left(L^{2}\right)}\left\|v_{2}\right\|_{L_{T^{\prime}}^{\infty}\left(L^{\infty}\right)}+C T^{\prime \frac{5}{4}}\|\nabla \delta \bar{v}\|_{L_{T^{\prime}}^{4}\left(L^{4}\right)}\left\|\partial_{t} \bar{v}_{2}\right\|_{L_{T^{\prime}}^{\infty}\left(L^{4}\right)} \\
& +C T^{\prime}\left\|\nabla \bar{v}_{1}\right\|_{L_{T^{\prime}}^{\infty}\left(L^{\infty}\right)}\left\|\partial_{t} \delta \bar{v}\right\|_{L_{T^{\prime}}^{2}\left(L^{2}\right)},
\end{aligned}
$$

where in the last inequality we have used the estimate that for every $t \in\left[0, T^{\prime}\right]$,

$$
\|\delta \bar{v}(t)\|_{L^{2}}=\|\delta \bar{v}(t)-\delta \bar{v}(0)\|_{L^{2}} \leq \int_{0}^{t}\left\|\partial_{\tau} \delta \bar{v}(\tau)\right\|_{L^{2}} \mathrm{~d} \tau \leq T^{\prime \frac{1}{2}}\left\|\partial_{t} \delta \bar{v}\right\|_{L_{T^{\prime}}^{2}\left(L^{2}\right)}
$$

Observing the magic equality that $\operatorname{div}\left(\left(A_{v_{1}}-A_{v_{2}}\right) \bar{v}_{2}\right)=\left(A_{v_{1}}^{\mathrm{T}}-A_{v_{2}}^{\mathrm{T}}\right): \nabla \bar{v}_{2}$ and $\operatorname{div}\left(\left(\mathrm{Id}-A_{v_{1}}\right) \delta \bar{v}\right)=\left(\operatorname{Id}-A_{v_{1}}^{\mathrm{T}}\right): \nabla \delta \bar{v}$ (e.g. see [7, Corollary A.3]), and by arguing as the estimation of $\mathrm{II}_{2}$, we deduce that

$$
\begin{aligned}
\mathrm{II}_{5} & \leq C T^{\prime}\left\|\left(\nabla \bar{v}_{1}, \nabla \bar{v}_{2}\right)\right\|_{L_{T^{\prime}}^{\infty}\left(L^{\infty}\right)}\left\|\nabla^{2} \delta \bar{v}\right\|_{L_{T^{\prime}}^{2}\left(L^{2}\right)} \\
& +C T^{\prime \frac{5}{4}}\left\|\left(\nabla^{2} \bar{v}_{1}, \nabla^{2} \bar{v}_{2}\right)\right\|_{L_{T^{\prime}}^{\infty}\left(L^{4}\right)}\|\nabla \delta \bar{v}\|_{L_{T^{\prime}}^{4}\left(L^{4}\right)} .
\end{aligned}
$$

Collecting (3.61), (3.63) and the above estimates on $\mathrm{II}_{2}-\mathrm{II}_{5}$, we obtain

$$
\begin{aligned}
\| \nabla \delta & \bar{v}\left\|_{L_{T^{\prime}}^{\infty}\left(L^{2}\right)}+\right\|\left(\partial_{t} \delta \bar{v}, \nabla^{2} \delta \bar{v}, \nabla \delta \bar{p}\right)\left\|_{L_{T^{\prime}}^{2}\left(L^{2}\right)}+\right\| \nabla \delta \bar{v} \|_{L_{T^{\prime}}^{4}\left(L^{4}\right)} \\
\leq & C T^{\prime}\left\|\nabla \bar{v}_{1}\right\|_{L_{T^{\prime}}^{\infty}\left(L^{\infty}\right)}\left\|\left(\partial_{t} \delta \bar{v}, \nabla \delta \bar{p}\right)\right\|_{L_{T^{\prime}}^{2}\left(L^{2}\right)}+C T^{\prime \frac{1}{2}}\left\|v_{2}\right\|_{L_{T^{\prime}}^{\infty}\left(L^{\infty}\right)}\|\nabla \delta \bar{v}\|_{L_{T^{\prime}}^{\infty}\left(L^{2}\right)} \\
& +C\left(T^{\prime}\left\|\left(\nabla \bar{v}_{1}, \nabla \bar{v}_{2}\right)\right\|_{L_{T^{\prime}}^{\infty}\left(L^{\infty}\right)}+T^{\prime \frac{5}{4}}\left\|\bar{\theta}_{2}\right\|_{L_{T^{\prime}}^{\infty}\left(L^{4}\right)}\right)\left\|\nabla^{2} \delta \bar{v}\right\|_{L_{T^{\prime}}^{2}\left(L^{2}\right)} \\
& +C T^{\prime \frac{5}{4}}\left\|\left(\bar{\theta}_{2}, \partial_{t} \bar{v}_{2}, \nabla \bar{p}_{2}, \nabla^{2} \bar{v}_{1}, \nabla^{2} \bar{v}_{2}\right)\right\|_{L_{T^{\prime}}^{\infty}\left(L^{4}\right)}\|\nabla \delta \bar{v}\|_{L_{T^{\prime}}^{4}\left(L^{4}\right)}
\end{aligned}
$$


where $C>0$ is a universal constant. Noting that uniform bounds (3.37) and (3.39) are at our disposal, we infer that by letting $T^{\prime}>0$ small enough so that

$$
\begin{aligned}
C T^{\prime}\left\|\left(\nabla \bar{v}_{1}, \nabla \bar{v}_{2}\right)\right\|_{L_{T}^{\infty}\left(L^{\infty}\right)}+C T^{\prime \frac{5}{4}}\left\|\bar{\theta}_{2}\right\|_{L_{T}^{\infty}\left(L^{4}\right)} \leq 1 / 2, \quad C T^{\prime \frac{1}{2}}\left\|v_{2}\right\|_{L_{T^{\prime}}^{\infty}\left(L^{\infty}\right)} \leq 1 / 2, \\
C T^{\prime \frac{5}{4}}\left\|\left(\bar{\theta}_{2}, \partial_{t} \bar{v}_{2}, \nabla \bar{p}_{2}, \nabla^{2} \bar{v}_{1}, \nabla^{2} \bar{v}_{2}\right)\right\|_{L_{T^{\prime}}^{\infty}\left(L^{4}\right)} \leq 1 / 2
\end{aligned}
$$

the whole right-hand side of (3.64) can be absorbed by the left-hand side, which yields $\|\nabla \delta \bar{v}\|_{L_{T^{\prime}}^{\infty}\left(L^{2}\right)}=\left\|\nabla^{2} \delta \bar{v}\right\|_{L_{T^{\prime}}^{2}\left(L^{2}\right)}=\|\nabla \delta \bar{v}\|_{L_{T^{\prime}}^{4}\left(L^{4}\right)} \equiv 0$, and also from estimate (3.61), $\|\delta \bar{\theta}\|_{L_{T^{\prime}}^{2}\left(L^{2}\right)} \equiv 0$. Since $\left.\delta \bar{v}\right|_{t=0}=0$, we conclude that $\delta \bar{v} \equiv 0$ and $\delta \bar{\theta} \equiv$ 0 on $\mathbb{R}^{2} \times\left[0, T^{\prime}\right]$. Repeating the above procedure and similarly as the treating of the corresponding part in [26], we can further prove $\delta \bar{v}=\delta \bar{\theta}=0$ on $\left[T^{\prime}, 2 T^{\prime}\right]$, $\left[2 T^{\prime}, 3 T^{\prime}\right], \ldots$, where $T^{\prime}>0$ is a small constant depending only on the time $T$ and the norms of $\left(\theta_{i}, v_{i}, p_{i}\right)$ in (3.36) (similar to conditions (3.38), (3.60), (3.65)), hence we finally get $\delta \bar{v}=\delta \bar{\theta} \equiv 0$ and also $X_{v_{1}}=X_{v_{2}}$ on $\mathbb{R}^{2} \times[0, T]$. Going back to the Eulerian coordinates implies that $\left(\mu_{1}, \theta_{1}, v_{1}\right)=\left(\mu_{2}, \theta_{2}, v_{2}\right)$ on $\mathbb{R}^{2} \times[0, T]$ and the uniqueness of system (1.3) immediately follows.

Acknowledgements P. B. Mucha was partially supported by National Science Centre grant No2018/29/B/ ST1/00339 (Opus). L. Xue was partially supported by National Natural Science Foundation of China (Grants Nos. 11671039 and 11771043$)$.

Open Access This article is licensed under a Creative Commons Attribution 4.0 International License, which permits use, sharing, adaptation, distribution and reproduction in any medium or format, as long as you give appropriate credit to the original author(s) and the source, provide a link to the Creative Commons licence, and indicate if changes were made. The images or other third party material in this article are included in the article's Creative Commons licence, unless indicated otherwise in a credit line to the material. If material is not included in the article's Creative Commons licence and your intended use is not permitted by statutory regulation or exceeds the permitted use, you will need to obtain permission directly from the copyright holder. To view a copy of this licence, visit http://creativecommons.org/licenses/by/4.0/.

\section{Appendix}

In this section we give the detailed proof of auxiliary Lemmas 2.5, 3.4 and 3.5.

Proof of Lemma 2.5 (1) For every $q \geq-1$, Bony's decomposition yields that

$$
\begin{aligned}
\Delta_{q}(v \cdot \nabla \theta)= & \sum_{k \in \mathbb{N},|k-q| \leq 4} \Delta_{q}\left(S_{k-1} v \cdot \nabla \Delta_{k} \theta\right)+\sum_{k \in \mathbb{N},|k-q| \leq 4} \Delta_{q}\left(\Delta_{k} v \cdot \nabla S_{k-1} \theta\right) \\
& +\sum_{k \geq-1, k \geq q-3} \Delta_{q}\left(\Delta_{k} v \cdot \nabla \widetilde{\Delta}_{k} \theta\right):=I_{q}+I I_{q}+I I I_{q}
\end{aligned}
$$

where $\Delta_{k}$ and $S_{k-1}$ are Littlewood-Paley operators introduced in (2.2) and $\widetilde{\Delta}_{k}:=$ $\Delta_{k-1}+\Delta_{k}+\Delta_{k+1}$. Taking advantage of Hölder's inequality, Bernstein's inequality 
and the fact that $\|v\|_{L^{2}}^{2}=\sum_{k \geq-1}\left\|\Delta_{k} v\right\|_{L^{2}}^{2}$, we get

$$
\begin{aligned}
& 2^{-q s}\left\|I_{q}\right\|_{L^{p}\left(\mathbb{R}^{2}\right)} \leq C_{0} \sum_{k \in \mathbb{N},|k-q| \leq 4} 2^{-q s}\left\|S_{k-1} v\right\|_{L^{\infty}\left(\mathbb{R}^{2}\right)}\left\|\nabla \Delta_{k} \theta\right\|_{L^{p}\left(\mathbb{R}^{2}\right)} \\
& \leq C_{0} \sum_{k \in \mathbb{N},|k-q| \leq 4}\left(\sum_{-1 \leq l \leq k-2}\left\|\Delta_{l} v\right\|_{L^{\infty}\left(\mathbb{R}^{2}\right)}\right) 2^{k(1-s)}\left\|\Delta_{k} \theta\right\|_{L^{p}\left(\mathbb{R}^{2}\right)} \\
& \quad \leq C_{0} \sum_{k \in \mathbb{N},|k-q| \leq 4}\left(\left\|\Delta_{-1} v\right\|_{L^{2}\left(\mathbb{R}^{2}\right)}+\sum_{0 \leq l \leq k+2} 2^{l}\left\|\Delta_{l} v\right\|_{L^{2}\left(\mathbb{R}^{2}\right)}\right) 2^{k(1-s)}\left\|\Delta_{k} \theta\right\|_{L^{p}\left(\mathbb{R}^{2}\right)} \\
& \quad \leq C_{0} \sum_{k \in \mathbb{N},|k-q| \leq 4}\left(\|v\|_{L^{2}\left(\mathbb{R}^{2}\right)}+\left(\sum_{0 \leq l \leq k+2}\left\|\Delta_{l} \nabla v\right\|_{L^{2}\left(\mathbb{R}^{2}\right)}^{2}\right)\right. \\
& \left.\quad \sqrt{k+2} 2^{k(1-s)}\left\|\Delta_{k} \theta\right\|_{L^{p}\left(\mathbb{R}^{2}\right)}\right) \\
& \leq C_{0}\left(\|v\|_{L^{2}\left(\mathbb{R}^{2}\right)}+\|\nabla v\|_{L^{2}\left(\mathbb{R}^{2}\right)}\right)\left(\sup _{k \geq-1} 2^{k(1-s)} \sqrt{k+2}\left\|\Delta_{k} \theta\right\|_{L^{p}\left(\mathbb{R}^{2}\right)}\right)
\end{aligned}
$$

and

$$
\begin{aligned}
2^{-q s}\left\|I I_{q}\right\|_{L^{p}\left(\mathbb{R}^{2}\right)} \leq & C_{0} \sum_{k \in \mathbb{N},|k-q| \leq 4} 2^{-q s}\left\|\Delta_{k} v\right\|_{L^{\infty}\left(\mathbb{R}^{2}\right)}\left\|\nabla S_{k-1} \theta\right\|_{L^{p}\left(\mathbb{R}^{2}\right)} \\
\leq & C_{0} \sum_{k \in \mathbb{N},|k-q| \leq 4}\left(\left\|\Delta_{-1} v\right\|_{L^{\infty}\left(\mathbb{R}^{2}\right)}+\left\|\left(\mathrm{Id}-\Delta_{-1}\right) \Delta_{k} v\right\|_{L^{\infty}\left(\mathbb{R}^{2}\right)}\right) \\
& \left(2^{-k s} \sum_{-1 \leq l \leq k-2} 2^{l}\left\|\Delta_{l} \theta\right\|_{L^{p}\left(\mathbb{R}^{2}\right)}\right) \\
\leq & C_{0} \sum_{k \in \mathbb{N},|k-q| \leq 4}\left(\|v\|_{L^{2}\left(\mathbb{R}^{2}\right)}+\left\|\left(\mathrm{Id}-\Delta_{-1}\right) \Delta_{k} \nabla v\right\|_{L^{2}\left(\mathbb{R}^{2}\right)}\right) \\
& \left(2^{-k s} \sum_{-1 \leq l \leq k-2} 2^{l s}\|\theta\|_{B_{p, \infty}^{1-s}\left(\mathbb{R}^{2}\right)}\right) \\
\leq & C\left(\|v\|_{L^{2}\left(\mathbb{R}^{2}\right)}+\|\nabla v\|_{L^{2}\left(\mathbb{R}^{2}\right)}\right)\|\theta\|_{B_{p, \infty}^{1-s}\left(\mathbb{R}^{2}\right)} .
\end{aligned}
$$

By using the divergence-free property of $v$, we similarly obtain

$$
\begin{aligned}
& 2^{-q s}\left\|I I I_{q}\right\|_{L^{p}\left(\mathbb{R}^{2}\right)} \\
& \quad \leq C_{0} \sum_{k \geq-1, k \geq q-3} 2^{q(1-s)}\left\|\Delta_{k} v\right\|_{L^{\infty}\left(\mathbb{R}^{2}\right)}\left\|\widetilde{\Delta}_{k} \theta\right\|_{L^{p}\left(\mathbb{R}^{2}\right)} \\
& \quad \leq C_{0}\left(\|v\|_{L^{2}\left(\mathbb{R}^{2}\right)}+\|\nabla v\|_{L^{2}\left(\mathbb{R}^{2}\right)}\right) \sum_{k \geq-1, k \geq q-3} 2^{(q-k)(1-s)} 2^{k(1-s)}\left\|\widetilde{\Delta}_{k} \theta\right\|_{L^{p}\left(\mathbb{R}^{2}\right)}
\end{aligned}
$$




$$
\leq C\left(\|v\|_{L^{2}\left(\mathbb{R}^{2}\right)}+\|\nabla v\|_{L^{2}\left(\mathbb{R}^{2}\right)}\right)\|\theta\|_{B_{p, \infty}^{1-s}\left(\mathbb{R}^{2}\right)} .
$$

Gathering the above estimates leads to (2.8), as desired.

(2) In order to prove the first inequality of (2.9), we also use the splitting (4.1), and by arguing as above, we deduce that for every $s>0$ and $q \geq-1$,

$$
\begin{aligned}
2^{q s}\left\|I_{q}\right\|_{L^{p}\left(\mathbb{R}^{2}\right)} & \leq C_{0} \sum_{k \in \mathbb{N},|k-q| \leq 4} 2^{q s}\left\|S_{k-1} v\right\|_{L^{2 p}\left(\mathbb{R}^{2}\right)}\left\|\nabla \Delta_{k} \theta\right\|_{L^{2 p}\left(\mathbb{R}^{2}\right)} \\
& \leq C_{0}\|v\|_{L^{2 p}\left(\mathbb{R}^{2}\right)}\|\nabla \theta\|_{B_{2 p, \infty}^{s}\left(\mathbb{R}^{2}\right)}, \\
2^{q s}\left\|I I_{q}\right\|_{L^{p}\left(\mathbb{R}^{2}\right)} & \leq C_{0} \sum_{k \in \mathbb{N},|k-q| \leq 4} 2^{q s}\left\|\Delta_{k} v\right\|_{L^{2 p}\left(\mathbb{R}^{2}\right)}\left\|S_{k-1} \nabla \theta\right\|_{L^{2 p}\left(\mathbb{R}^{2}\right)} \\
& \leq C\|v\|_{B_{2 p, \infty}^{s}\left(\mathbb{R}^{2}\right)}\|\nabla \theta\|_{L^{2 p}\left(\mathbb{R}^{2}\right)}, \\
2^{q s}\left\|I I I_{q}\right\|_{L^{p}\left(\mathbb{R}^{2}\right)} & \leq C_{0} \sum_{k \geq-1, k \geq q-3} 2^{q s}\left\|\Delta_{k} v\right\|_{L^{2 p}\left(\mathbb{R}^{2}\right)}\left\|\widetilde{\Delta}_{k} \nabla \theta\right\|_{L^{2 p}\left(\mathbb{R}^{2}\right)} \\
& \leq C_{0}\left(\sum_{k \geq-1, k \geq q-3} 2^{(q-k) s}\|v\|_{B_{2 p, \infty}^{s}\left(\mathbb{R}^{2}\right)}\|\nabla \theta\|_{L^{2 p}\left(\mathbb{R}^{2}\right)}\right) \\
& \leq C\|v\|_{B_{2 p, \infty}^{s}\left(\mathbb{R}^{2}\right)}\|\nabla \theta\|_{L^{2 p}\left(\mathbb{R}^{2}\right)} .
\end{aligned}
$$

Hence, by collecting the above estimates we conclude the first inequality of (2.9), and then from continuous embedding $B_{2 p, \infty}^{s} \hookrightarrow L^{2 p}$ the second inequality of (2.9) directly follows.

Proof of Lemma 3.4 We here only focus on the a priori estimates of $w$, and the existence and uniqueness of weak solution can be done by a standard approximation process. By using the equation (3.49) and the integration by parts, it follows that

$$
\begin{aligned}
\int_{\mathbb{R}^{2}}|\delta \bar{\theta}|^{2} \mathrm{~d} x=\int_{\mathbb{R}^{2}}\left|\partial_{t} w+\Delta w\right|^{2} \mathrm{~d} x= & \int_{\mathbb{R}^{2}}\left|\partial_{t} w\right|^{2} \mathrm{~d} x+\int_{\mathbb{R}^{2}}|\Delta w|^{2} \mathrm{~d} x \\
& +2 \int_{\mathbb{R}^{2}} \partial_{t} w \Delta w \mathrm{~d} x \\
= & \int_{\mathbb{R}^{2}}\left|\partial_{t} w\right|^{2} \mathrm{~d} x+\int_{\mathbb{R}^{2}}\left|\nabla^{2} w\right|^{2} \mathrm{~d} x \\
& -\frac{\mathrm{d}}{\mathrm{d} t} \int_{\mathbb{R}^{2}}|\nabla w|^{2} \mathrm{~d} x
\end{aligned}
$$

Integrating on the time interval $\left[t, T^{\prime}\right]$ (for every $t \in\left[0, T^{\prime}\right]$ ) leads to

$$
\sup _{t \in\left[0, T^{\prime}\right]}\|\nabla w(t)\|_{L^{2}}^{2}+\left\|\nabla^{2} w\right\|_{L^{2}\left(0, T^{\prime} ; L^{2}\right)}^{2}+\left\|\partial_{t} w\right\|_{L^{2}\left(0, T^{\prime} ; L^{2}\right)}^{2} \leq 2\|\delta \bar{\theta}\|_{L^{2}\left(0, T^{\prime} ; L^{2}\right)}^{2} .
$$

Combined with interpolation inequality (3.62), we get $\|\nabla w\|_{L^{4}\left(0, T^{\prime} ; L^{4}\right)}$ $\leq C_{0}\|\delta \bar{\theta}\|_{L^{2}\left(0, T^{\prime} ; L^{2}\right)}$ and the desired estimate (3.50). On the other hand, by taking 
the scalar product of Eq. (3.49) with $w$, we have

$$
-\frac{1}{2} \frac{\mathrm{d}}{\mathrm{d} t}\|w(t)\|_{L^{2}}^{2}+\int_{\mathbb{R}^{2}}|\nabla w|^{2} \mathrm{~d} x \leq\|w(t)\|_{L^{2}}\|\delta \bar{\theta}(t)\|_{L^{2}}
$$

which implies that $-\frac{\mathrm{d}}{\mathrm{d} t}\|w(t)\|_{L^{2}} \leq\|\delta \bar{\theta}\|_{L^{2}}$, and then integrating on the time interval $\left[t, T^{\prime}\right]$ yields $\|w\|_{L^{\infty}\left(0, T^{\prime} ; L^{2}\right)} \leq T^{\prime \frac{1}{2}}\|\delta \bar{\theta}\|_{L^{2}\left(0, T^{\prime} ; L^{2}\right)}$. Hence, the solution $w$ belongs to $L^{\infty}\left(0, T^{\prime} ; H^{1}\right) \cap L^{2}\left(0, T^{\prime} ; H^{2}\right)$.

Proof of Lemma 3.5 Denoting by $C_{v_{i}}(t, y)=\int_{0}^{t} \nabla \bar{v}_{i}(\tau, y) \mathrm{d} y, i=1,2$, and using expression formula (2.24), it is easy to see that

$$
\begin{aligned}
A_{v_{1}}-A_{v_{2}}= & \sum_{k=1}^{\infty}(-1)^{k}\left(C_{v_{1}}^{k}-C_{v_{2}}\right)^{k}=\sum_{k=1}^{\infty} \sum_{j=0}^{k-1}(-1)^{k} C_{v_{1}}^{j} C_{v_{2}}^{k-1-j} \int_{0}^{t} \nabla \delta \bar{v} \mathrm{~d} \tau, \\
\nabla A_{v_{i}}= & \sum_{k=0}^{\infty}(-1)^{k+1}(k+1) C_{v_{i}}^{k} \int_{0}^{t} \nabla^{2} \bar{v}_{i} \mathrm{~d} \tau, \\
\nabla A_{v_{1}}-\nabla A_{v_{2}}= & \sum_{k=0}^{\infty}(-1)^{k+1}(k+1) C_{v_{2}}^{k} \int_{0}^{t} \nabla^{2} \delta \bar{v} \mathrm{~d} \tau \\
& +\sum_{k=1}^{\infty} \sum_{j=0}^{k-1}(-1)^{k+1}(k+1) C_{v_{1}}^{j} C_{v_{2}}^{k-1-j}\left(\int_{0}^{t} \nabla \delta \bar{v} \mathrm{~d} \tau\right) \int_{0}^{t} \nabla^{2} \bar{v}_{1} \mathrm{~d} \tau, \\
\partial_{t} A_{v_{i}}(t, y)= & \sum_{k=1}^{\infty}(-1)^{k} k C_{v_{i}}^{k-1} \nabla \bar{v}_{i}(t, y), \\
\partial_{t} A_{v_{1}}-\partial_{t} A_{v_{2}}= & (\nabla \delta \bar{v}) \sum_{k=1}^{\infty}(-1)^{k} k C_{v_{2}}^{k-1}+\nabla \bar{v}_{1} \sum_{k=2}^{\infty} \sum_{j=0}^{k-2}(-1)^{k} k C_{v_{1}}^{j} C_{v_{2}}^{k-2-j} \int_{0}^{t} \nabla \delta \bar{v} \mathrm{~d} \tau,
\end{aligned}
$$

with $\delta \bar{v}:=\bar{v}_{1}-\bar{v}_{2}$. Hence, by using Hölder's inequality and condition (3.52), estimates (3.53)-(3.59) directly follow from (2.24) and the above expression formulas.

\section{References}

1. Agroum, R.: A posteriori error analysis for solving the Navier-Stokes problem and convection-diffusion equation. Numer. Methods Partial Differ. Equ. 34(2), 401-418 (2018)

2. Bahouri, H., Chemin, J.-Y., Danchin, R.: Fourier Analysis and Nonlinear Partial Differential Equations. Springer, Heidelberg (2011)

3. Brandolese, L., Schonbek, M.E.: Large time decay and growth for solutions of a viscous Boussinesq system. Trans. Am. Math. Soc. 364(10), 5057-5090 (2012)

4. Cannon, J.R., DiBenedetto, E.: The initial problem for the Boussinesq equations with data in $L^{p}$. Lecture Note in Mathematics, vol. 771, pp. 129-144. Springer, Berlin (1980)

5. Danchin, R.: Global existence in critical spaces for compressible Navier-Stokes equations. Invent. Math. 141, 579-614 (2000)

6. Danchin, R.: Fourier analysis methods for PDE's. unpublished notes (2005). http://perso-math.univmlv.fr/users/danchin.raphael/cours/courschine.pdf

7. Danchin, R., Mucha, P.B.: A Lagrangian approach for the incompressible Navier-Stokes equation with variable density. Commun. Pure Appl. Math. 65, 1458-1480 (2012) 
8. Danchin, R., Mucha, P.B.: Incompressible flows with piecewise constant density. Arch. Ration. Mech. Anal. 207, 991-1023 (2013)

9. Danchin, R., Mucha, P.B.: Critical functional framework and maximal regularity in action on systems of incompressible flows. Mém. Soc. Math. Fr. (N.S.) No. 143 (2015)

10. Danchin, R., Mucha, P.B.: Compressible Navier-Stokes system: large solutions and incompressible limit. Adv. Math. 320, 904-925 (2017)

11. Deteix, J., Jendoubi, A., Yakoubi, D.: A coupled prediction scheme for solving the Navier-Stokes and convection-diffusion equations. SIAM J. Numer. Anal. 52(5), 2415-2439 (2014)

12. Feireisl, E.: Dynamics of Viscous Compressible Fluids. Oxford Lecture Series in Mathematics and its Applications, vol. 26. Oxford (2004)

13. Feireisl, E., Mucha, P.B., Novotny, A., Pokorny, M.: Time-periodic solutions to the full Navier-StokesFourier system. Arch. Ration. Mech. Anal. 204(3), 745-786 (2012)

14. Feireisl, E., Novotny, A.: Singular limits in thermodynamics of viscous fluids. Advances in Mathematical Fluid Mechanics. Birkhäuser Verlag (2009)

15. Feireisl, E., Novotny, A.: The Oberbeck-Boussinesq approximation as a singular limit of the full Navier-Stokes-Fourier system. J. Math. Fluid Mech. 11(2), 274-302 (2009)

16. Giga, Y., Miyakawa, T., Osada, H.: Two-dimensional Navier-Stokes flow with measures as initial vorticity. Commun. Math. Phys. 104, 223-250 (1988)

17. Guo, B.: Spectral method for solving two-dimensional Newton-Boussinesq equation. Acta Math. Appl. Sin. 5, 208-218 (1989)

18. Gwiazda, P., Lorenz, T., Marciniak-Czochra, A.: A nonlinear structured population model: Lipchitz continuity of measure-valued solutions with respect to model ingredients. J. Differ. Equ. 248, 27032735 (2010)

19. Hmidi, T., Keraani, S., Rousset, F.: Global well-posedness for a Boussinesq-Navier-Stokes system with critical dissipation. J. Differ. Equ. 249(9), 2147-2174 (2010)

20. Hmidi, T., Rousset, F.: Global well-posedness for the Navier-Stokes-Boussinesq system with axisymmetric data. Ann. Inst. H. Poincaré Anal. Non Linéaire 27(5), 1227-1246 (2010)

21. Hsia, Ch-H., Lin, Ch-Sh., Ma, T., Wang, S.: Tropical atmospheric circulations with humidity effects. Proc. A. 471(2173), 20140353 (2015)

22. Ladyzhenskaya, O.A.: Solution 'in the large' of the non-stationary boundary value problem for the Navier-Stokes system with two space variables. Commun. Pure Appl. Math. 12, 427-433 (1959)

23. Li, D., Rodrigo, J.: Wellposedness and regularity of solutions of an aggregation equation. Rev. Mat. Iberoam. 26(1), 261-294 (2010)

24. Matsumura, A., Nishida, T.: Initial boundary value problem for equations of motion of compressible viscous and heat conductive fluids. Commun. Math. Phys. 89, 445-464 (1983)

25. Mucha, P.B., Peszek, J.: The Cucker-Smale equation: singular communication weight, measure-valued solutions and weak-atomic uniqueness. Arch. Ration. Mech. Anal. 227, 273-308 (2018)

26. Mucha, P.B., Xue, L., Zheng, X.: Between homogeneous and inhomogeneous Navier-Stokes systems: the issue of stability. J. Differ. Equ. 267(1), 307-363 (2019)

27. Paicu, M., Zhang, P., Zhang, Z.: Global unique solvability of inhomogeneous Navier-Stokes equations with bounded density. Commun. Partial Differ. Equ. 38, 1208-1234 (2013)

28. Temam, R.: Navier-Stokes Equations. Theory and Numerical Analysis. North-Holland, Amsterdam (1984)

29. Valli, A., Zajaczkowski, W.M.: Navier-Stokes equations for compressible fluids: global existence and qualitative properties of the solutions in the general case. Commun. Math. Phys. 103(2), 259-296 (1986)

Publisher's Note Springer Nature remains neutral with regard to jurisdictional claims in published maps and institutional affiliations. 